\title{
INHOMOGENEOUS TORSIONAL CREEP PROBLEMS IN ANISOTROPIC ORLICZ SOBOLEV SPACES
}

\author{
MIHAI MIHĂILESCU \& MAYTE PÉREZ-LLANOS
}

\begin{abstract}
In this paper we study the asymptotic behavior of the sequence of solutions for a family of torsional creep-type problems, involving inhomogeneous and anisotropic differential operators, on a bounded domain, subject to the homogenous Dirichlet boundary condition. We find out that the sequence of solutions converges uniformly on the domain to a certain distance function defined in accordance with the anisotropy of the problem. In addition, we identify the limit problem via viscosity solutions theory.
\end{abstract}

\section{INTRODUCTION}

Let $L, M$ and $N$ be three positive integers such that $L+M=N$. For each $\xi \in \mathbb{R}^{N}$ we write $\xi=(x, y) \in \mathbb{R}^{L} \times \mathbb{R}^{M}$ with $x=\left(x_{1}, \ldots, x_{L}\right) \in \mathbb{R}^{L}$ and $y=$ $\left(y_{1}, \ldots, y_{M}\right) \in \mathbb{R}^{M}$. Moreover, we denote by $|\cdot|_{L},|\cdot|_{M}$ and $|\cdot|_{N}$, the Euclidean norms in $\mathbb{R}^{L}, \mathbb{R}^{M}$ and $\mathbb{R}^{N}$, respectively. Furthermore, for $\xi_{1}=(\bar{x}, \bar{y}) \in \mathbb{R}^{N}$ and $\xi_{2}=(\tilde{x}, \tilde{y}) \in \mathbb{R}^{N}$ with $\bar{x}, \tilde{x} \in \mathbb{R}^{L}$ and $\bar{y}, \tilde{y} \in \mathbb{R}^{M}$ we define the "anisotropic Euclidean norm" on $\mathbb{R}^{N}$ as

$$
] \xi_{1}-\xi_{2}\left[{ }_{1}:=|\bar{x}-\tilde{x}|_{L}+|\bar{y}-\tilde{y}|_{M} .\right.
$$

On the other hand, for a sufficiently smooth function $u$ defined on an open subset of $\mathbb{R}^{N}$ we will use the following notations

$$
\nabla_{x} u:=\left(\frac{\partial u}{\partial x_{1}}, \ldots, \frac{\partial u}{\partial x_{L}}\right), \quad \nabla_{y} u:=\left(\frac{\partial u}{\partial y_{1}}, \ldots, \frac{\partial u}{\partial y_{M}}\right), \quad \nabla u:=\left(\nabla_{x} u, \nabla_{y} u\right) .
$$

For each integer $n \geq 1$, we consider the mappings $\phi_{n}, \psi_{n}: \mathbb{R} \rightarrow \mathbb{R}$ which are assumed to be odd, increasing homeomorphisms of class $C^{1}$ satisfying

$$
\begin{array}{ll}
N-1<\phi_{n}^{-}-1 \leq \frac{t \phi_{n}^{\prime}(t)}{\phi_{n}(t)} \leq \phi_{n}^{+}-1<\infty, & \forall t \geq 0 \\
N-1<\psi_{n}^{-}-1 \leq \frac{t \psi_{n}^{\prime}(t)}{\psi_{n}(t)} \leq \psi_{n}^{+}-1<\infty, \quad \forall t \geq 0
\end{array}
$$

for some constants $\phi_{n}^{-}, \psi_{n}^{-}, \phi_{n}^{+}, \psi_{n}^{+}$with $N<\phi_{n}^{-} \leq \phi_{n}^{+}<\infty$ and $N<\psi_{n}^{-} \leq \psi_{n}^{+}<$ $\infty$

$$
\phi_{n}^{-} \rightarrow \infty \text { and } \psi_{n}^{-} \rightarrow \infty \text { as } n \rightarrow \infty,
$$

and such that there exists a real constant $\beta>1$ for which

$$
\phi_{n}^{+} \leq \beta \phi_{n}^{-} \text {and } \psi_{n}^{+} \leq \beta \psi_{n}^{-}, \quad \forall n \geq 1 .
$$

Key words and phrases. anisotropic operator; Orlicz-Sobolev space; $\Gamma$-convergence; viscosity solution.

2010 Mathematics Subject Classification. 35D30; 35D40; 46E30; 49J40. 
Let $\Omega \subset \mathbb{R}^{N}$ be a bounded domain with smooth boundary, $\partial \Omega$, and let $f \in$ $C(\bar{\Omega})$ be a positive function. The purpose of this work is to study the asymptotic behaviour of the solutions for the family of problems

$$
\begin{cases}-\operatorname{div}_{x}\left(\frac{\phi_{n}\left(\left|\nabla_{x} u\right|_{L}\right)}{\phi_{n}(1)\left|\nabla_{x} u\right|_{L}} \nabla_{x} u\right)-\operatorname{div}_{y}\left(\frac{\psi_{n}\left(\left|\nabla_{y} u\right|_{M}\right)}{\psi_{n}(1)\left|\nabla_{y} u\right|_{M}} \nabla_{y} u\right)=f, & \text { in } \Omega, \\ u=0, & \text { on } \partial \Omega,\end{cases}
$$

as $n \rightarrow \infty$.

Several research directions are assembled behind the study of the present work. The initial motivation comes from the analysis of torsional creep problems. According to [4] the torsional creep represents the permanent plastic deformation of a material subject to a torsional moment for an extended period of time and at sufficiently high temperature. Moreover, in [4, Part IV] it is also pointed out the fact that torsional creep problems are related to inhomogeneous problems of the type

$$
\begin{cases}-\operatorname{div}\left(|\nabla u|_{N}^{p-2} \nabla u\right)=1 & \text { in } \Omega, \\ u=0 & \text { on } \partial \Omega .\end{cases}
$$

It is common knowledge that for all real numbers $p \in(1, \infty)$ problem $(1.6)$ possesses a unique solution, say $u_{p}$. As explained in [23], several facts on elastic-plastic torsion theory suggested that necessarily $u_{p} \rightarrow \operatorname{dist}(\cdot, \partial \Omega)$ in some sense, where $\operatorname{dist}(\cdot, \partial \Omega)$ stands for the distance function to the boundary of $\Omega$ with respect to the Euclidean $\operatorname{norm}|\cdot|_{N}$, i.e. $\operatorname{dist}(x, \partial \Omega):=\inf _{y \in \partial \Omega}|x-y|_{N}$, for each $x \in \Omega$. One of the first results supporting this conjecture is included in [26], where it is shown that

$$
\lim _{p \rightarrow \infty} \int_{\Omega} u_{p}(x) d x \rightarrow \int_{\Omega} \operatorname{dist}(x, \partial \Omega) d x .
$$

This result was improved independently in [23] and [4] where it was established the uniform convergence of $u_{p}$ to $\operatorname{dist}(\cdot, \partial \Omega)$ in $\bar{\Omega}$. If in [23] the uniform convergence was obtained with the use of variational arguments and maximum principles, in [4] the authors used an approach based on the analysis of the viscosity solutions of the limiting problem of the family of equations (1.6), as $p \rightarrow \infty$. In order to explain the ideas used in [4] we recall the famous Lipschitz Extension Problem, that is, given a Lipschitz function $g: \partial \Omega \rightarrow \mathbb{R}$, with its best Lipschitz constant $L_{g}$, find its Absolutely Minimizing Lipschitz Extension (or $A M L E$ ), i.e. the (unique) best Lipschitz extension, in the sense that the Lipschitz constant is the same, $L_{g}$, and it remains stable for subdomains (see [2]). The problem of finding the $A M L E$ can be connected with the study of the limit problem, as $p \rightarrow \infty$, of the family of equations $\operatorname{div}\left(|\nabla u|_{N}^{p-2} \nabla u\right)=0$ in $\Omega$ subject to $u=g$ on $\partial \Omega$. This idea gave rise to a notable field of research, where the limit problem is identified and the existence of solutions for the limit problem is investigated, thanks to a relaxed concept of solution: solutions in viscosity sense, see Section 6 for further details on this topic. In this context, we recall that the limit problem corresponding to (1.6), as $p \rightarrow \infty$, reads as $\min \left\{1-|\nabla u|_{N},-\Delta_{\infty} u\right\}=0$ in $\Omega$ and $u=0$ on $\partial \Omega$, which has a unique (viscosity) solution that is precisely $\operatorname{dist}(\cdot, \partial \Omega)$ (see, e.g. $[18,20]$ ). Here $\Delta_{\infty} u:=\left\langle D^{2} u \nabla u, \nabla u\right\rangle_{N}$ stands for the Infinity Laplacian, provided that $D^{2} u$ denotes the Hessian matrix of $u$ and $\langle\cdot, \cdot\rangle_{N}$ is the scalar product on $\mathbb{R}^{N}$. 
The above results can be relaxed in the sense that we can consider more general differential operators in the left-hand side of equations (1.6). For example, in [29] the authors replaced the $p$-Laplacian from (1.6), i.e. $\operatorname{div}\left(|\nabla u|_{N}^{p-2} \nabla u\right)$, by its inhomogeneous variable exponent version, the $p(\cdot)$-Laplacian, i.e. $\operatorname{div}\left(|\nabla u|_{N}^{p(x)-2} \nabla u\right)$, where $p: \bar{\Omega} \rightarrow(1, \infty)$ is a continuous function. To be more specific, it was proved that if $p_{n}(\cdot)$ is a sequence of continuous functions over $\Omega$ which diverges to infinity uniformly in $\Omega$, as $n \rightarrow \infty$, then the sequence of (unique) solutions, $u_{n}$, of the family of equations

$$
\begin{cases}-\operatorname{div}\left(|\nabla u|_{N}^{p_{n}(x)-2} \nabla u\right)=1, & \text { in } \Omega \\ u=0, & \text { on } \partial \Omega\end{cases}
$$

converges uniformly to $\operatorname{dist}(\cdot, \partial \Omega)$ in $\bar{\Omega}$, as $n \rightarrow \infty$. Another generalization of (1.6) is undertaken in [6], where the authors studied the asymptotic behavior of the sequence of (unique) solutions, $u_{n}$, for the family of problems

$$
\begin{cases}-\operatorname{div}\left(\frac{\phi_{n}\left(|\nabla u|_{N}\right)}{\phi_{n}(1)|\nabla u|_{N}} \nabla u\right)=1, & \text { in } \Omega, \\ u=0, & \text { on } \partial \Omega,\end{cases}
$$

with $\phi_{n}$ satisfying (1.1), (1.3) and (1.4). It was established again that $u_{n}$ converges uniformly to $\operatorname{dist}(\cdot, \partial \Omega)$ in $\bar{\Omega}$, as $n \rightarrow \infty$. Note that, problems (1.7) and (1.8) represent generalizations of (1.6) since if we consider the particular cases when $p_{n}(x)=n$ and $\phi_{n}(t)=|t|^{n-2} t$, respectively, we recover (1.6) with $p=n$. On the other hand, we also point out the fact that problem (1.8) can be regarded as an isotropic version of problem (1.5), proposed for investigation in this paper. However, as it can be easily observed, we can not apply the results obtained from the study of problems (1.8) to the study of problems (1.5) since the differential operator involved in (1.5) is of anisotropic type. As we will show immediately this new situation will give rise some changes in the conclusion of the investigation considered here compared with the conclusion of the isotropic case.

We recall that the analysis of asymptotic behavior of solutions for different families of anisotropic differential operators was largely considered in the literature, see for instance $[3,14,16,27,29,28]$. In particular, for each positive integer $n$, consider the family of equations

$$
\begin{cases}-\operatorname{div}_{x}\left(\left|\nabla_{x} u_{n}\right|_{L}^{p_{n}-2} \nabla_{x} u_{n}\right)-\operatorname{div}_{y}\left(\left|\nabla_{y} u_{n}\right|_{M}^{q_{n}-2} \nabla_{y} u_{n}\right)=1, & \text { in } \Omega, \\ u_{n}=0, & \text { on } \partial \Omega,\end{cases}
$$

where $p_{n}$ and $q_{n}$ are two sequences of real numbers diverging to infinity, as $n \rightarrow \infty$. For each integer $n$ we denote by $u_{n}$ the unique solution of (1.9). It was proved in [14] and [16, Section 6] (see also [27] for a variable exponent version of the problem) that $u_{n}$ converges uniformly in $\Omega$, as $n \rightarrow \infty$, to a distance function that takes into account the anisotropy of the problem, see Section 3. Indeed it is the unique solution of the limit problem, identified to be precisely $\max \left\{\left|\nabla_{x} u\right|_{L},\left|\nabla_{y} u\right|_{M}\right\}=1$ in $\Omega$ subjected to $u=0$ on $\partial \Omega$. Since problem (1.9) represents a particular case of problem (1.5), obtained when $\phi_{n}=|t|^{p_{n}-2} t$ and $\psi_{n}=|t|^{q_{n}-2} t$, our intuition is that we can expect to prove similar results in the case of problem (1.5). 
The goal of the present paper is to complement all the former works highlighted above by analyzing problem (1.5), which, due to its anisotropic nature, could represent a torsion that twists the material depending on the direction of the variables.

The paper is organized as follows: in Section 2 we present the function space setting where problem (1.5) is analyzed; in Section 3 the definition and properties of a weak solution for problem (1.5) are recalled and the main result of this paper is emphasized; Section 4 is devoted to the study of a $\Gamma$-convergence result for certain functionals which are related with the problem considered here; subsequently, this $\Gamma$-convergence result is used in Section 5 in order to prove the main result of the paper; Section 6 presents an alternative proof of the main result based on the identification of the limit equation, as $n \rightarrow \infty$ in (1.5), which is understood in the viscosity sense.

\section{FunCtion SPACE SETting}

Due to the inhomogeneous nature of the problem (1.5), the classical Lebesgue and Sobolev spaces are not the appropriate functional spaces in which to seek solutions for our problem. Instead, one needs to work in the more general framework of Orlicz and Orlicz-Sobolev spaces. With $\phi_{n}$ and $\psi_{n}$ as above, define

$$
\Phi_{n}(t):=\int_{0}^{t} \phi_{n}(s) d s \text { and } \Psi_{n}(t):=\int_{0}^{t} \psi_{n}(s) d s, \quad \forall t>0 .
$$

Note that hypotheses (1.1) and (1.2) ensure that

$$
\phi_{n}^{-} \leq \frac{t \phi_{n}(t)}{\Phi_{n}(t)} \leq \phi_{n}^{+} \quad \text { and } \quad \psi_{n}^{-} \leq \frac{t \psi_{n}(t)}{\Psi_{n}(t)} \leq \psi_{n}^{+}, \quad \forall t \geq 0,
$$

as it is shown in [24, Lemma 1.1] (see also, [25, Lemma 2.1]). The Orlicz spaces $L^{\Phi_{n}}(\Omega)$ and $L^{\Psi_{n}}(\Omega)$ are defined by

$$
L^{\Phi_{n}}(\Omega):=\left\{u: \Omega \rightarrow \mathbb{R} ; \quad u \text { is measurable and } \int_{\Omega} \Phi_{n}(|u(\xi)|) d \xi<\infty\right\},
$$

and

$$
L^{\Psi_{n}}(\Omega):=\left\{u: \Omega \rightarrow \mathbb{R} ; \quad u \text { is measurable and } \int_{\Omega} \Psi_{n}(|u(\xi)|) d \xi<\infty\right\} .
$$

Endowed with the Luxemburg norms, given by

$$
\|u\|_{\Phi_{n}}:=\inf \left\{\mu>0: \int_{\Omega} \Phi_{n}\left(\frac{u(\xi)}{\mu}\right) d \xi \leq 1\right\},
$$

and

$$
\|u\|_{\Psi_{n}}:=\inf \left\{\mu>0: \int_{\Omega} \Psi_{n}\left(\frac{u(\xi)}{\mu}\right) d \xi \leq 1\right\},
$$

$L^{\Phi_{n}}(\Omega)$ and $L^{\Psi_{n}}(\Omega)$ are Banach spaces. The Orlicz-Sobolev space $W_{0}^{1, \Phi_{n}, \Psi_{n}}(\Omega)$ is defined as the closure of $C_{0}^{\infty}(\Omega)$ with respect to the norm $\|u\|:=\left\|\left|\nabla_{x} u\right|_{L}\right\|_{\Phi_{n}}+$ $\left\|\left|\nabla_{y} u\right|_{M}\right\|_{\Psi_{n}}$. Under our assumptions (1.1) and (1.2), $L^{\Phi_{n}}(\Omega), L^{\Psi_{n}}(\Omega)$ and $W_{0}^{1, \Phi_{n}, \Psi_{n}}(\Omega)$ are reflexive Banach spaces. For more details about Orlicz and Orlicz-Sobolev spaces we refer to the book by Adams [1], and to the papers by Clément et al. [9], Lieberman [24] and Martínez \& Wolanski [25]. 


\section{MAIn RESUlts}

We say that $u \in W_{0}^{1, \Phi_{n}, \Psi_{n}}(\Omega)$ is a weak solution of problem (1.5) if

$$
\int_{\Omega} \frac{\phi_{n}\left(\left|\nabla_{x} u\right|_{L}\right)}{\phi_{n}(1)\left|\nabla_{x} u\right|_{L}} \nabla_{x} u \nabla_{x} v d \xi+\int_{\Omega} \frac{\psi_{n}\left(\left|\nabla_{y} u\right|_{M}\right)}{\psi_{n}(1)\left|\nabla_{y} u\right|_{M}} \nabla_{y} u \nabla_{y} v d \xi=\int_{\Omega} f v d \xi,
$$

for all $v \in W_{0}^{1, \Phi_{n}, \Psi_{n}}(\Omega)$.

It is standard that for each positive integer $n \in \mathbb{N}$, the unique weak solution $u_{n} \in W_{0}^{1, \Phi_{n}, \Psi_{n}}(\Omega)$ of problem (1.5) is a minimizer of the Euler-Lagrange functional associated to problem (1.5), namely, $J_{n}: W_{0}^{1, \Phi_{n}, \Psi_{n}}(\Omega) \rightarrow \mathbb{R}$ given by

$$
J_{n}(v):=\int_{\Omega} \frac{\Phi_{n}\left(\left|\nabla_{x} v(\xi)\right|_{L}\right)}{\phi_{n}(1)} d \xi+\int_{\Omega} \frac{\Psi_{n}\left(\left|\nabla_{y} v(\xi)\right|_{M}\right)}{\psi_{n}(1)} d \xi-\int_{\Omega} f(\xi) v(\xi) d \xi
$$

In particular, since $J_{n}(v) \geq J_{n}(|v|)$ for all $v \in W_{0}^{1, \Phi_{n}, \Psi_{n}}(\Omega)$ and $u_{n}$ is a minimizer of $J_{n}$, it is clear that $u_{n}(\xi) \geq 0$ for a.e. $\xi \in \Omega$. Moreover, it follows by standard arguments that $J_{n} \in C^{1}\left(W_{0}^{1, \Phi_{n}, \Psi_{n}}(\Omega) ; \mathbb{R}\right)$ and $\left\langle J_{n}^{\prime}\left(u_{n}\right), u_{n}\right\rangle=0$, i.e.

$$
\begin{aligned}
\int_{\Omega} \frac{\phi_{n}\left(\left|\nabla_{x} u_{n}\right|_{L}\right)}{\phi_{n}(1)}\left|\nabla_{x} u_{n}\right|_{L} d \xi & +\int_{\Omega} \frac{\psi_{n}\left(\left|\nabla_{y} u_{n}\right|_{M}\right)}{\psi_{n}(1)}\left|\nabla_{y} u_{n}\right|_{M} d \xi \\
& -\int_{\Omega} f u_{n} d \xi=0 .
\end{aligned}
$$

Let us introduce the "anisotropic distance function" to the boundary of $\Omega$ with respect to the norm $] \cdot\left[{ }_{1}\right.$ as $\delta_{1}: \Omega \rightarrow[0, \infty)$, determined by

$$
\left.\delta_{1}(\xi)=\inf _{\eta \in \partial \Omega}\right] \xi-\eta[1, \quad \forall \xi \in \Omega .
$$

Our aim is to see that, indeed, this anisotropic distance will be the limit of our solutions $u_{n}$ as $n \rightarrow \infty$. This is the core of the following theorem.

Theorem 3.1. Assume that $f \in C(\bar{\Omega})$ is positive and the hypotheses (1.1), (1.2), (1.3), and (1.4) hold. Then the sequence $\left\{u_{n}\right\} \subset W_{0}^{1, \Phi_{n}, \Psi_{n}}(\Omega)$ of weak solutions of problem (1.5) converges uniformly in $\Omega$ to $\delta_{1}$.

\section{A $\Gamma$-CONVERGENCE RESUlt}

We dedicate this section of our paper to analyze the $\Gamma$-convergence of certain functionals which are strongly related with the problem under consideration.

Let us first recall the definition of the concept of $\Gamma$-convergence (introduced in [12], [13]) in metric spaces. The reader is referred to [11] and [7] for a comprehensive introduction to the topic.

Definition 4.1. Let $X$ be a metric space. A sequence $\left\{F_{n}\right\}$ of functionals $F_{n}$ : $X \rightarrow \overline{\mathbb{R}}:=\mathbb{R} \cup\{+\infty\}$ is said to $\Gamma(X)$-converge to $F: X \rightarrow \overline{\mathbb{R}}$, and we write $\Gamma(X)-\lim _{n \rightarrow \infty} F_{n}=F$, if the following hold:

(i): for every $u \in X$ and $\left\{u_{n}\right\} \subset X$ such that $u_{n} \rightarrow u$ in $X$, we have

$$
F(u) \leq \liminf _{n \rightarrow \infty} F_{n}\left(u_{n}\right) ;
$$

(ii): for every $u \in X$ there exists a recovery sequence $\left\{u_{n}\right\} \subset X$ such that $u_{n} \rightarrow u$ in $X$ and

$$
F(u) \geq \limsup _{n \rightarrow \infty} F_{n}\left(u_{n}\right)
$$


For each integer $n \geq 1$ consider the functional $I_{n}: L^{1}(\Omega) \rightarrow[0, \infty]$ defined by

$$
I_{n}(u)= \begin{cases}\int_{\Omega} \frac{\Phi_{n}\left(\left|\nabla_{x} u\right|_{L}\right)}{\phi_{n}(1)} d \xi+\int_{\Omega} \frac{\Psi_{n}\left(\left|\nabla_{y} u\right|_{M}\right)}{\psi_{n}(1)} d \xi, & \text { if } u \in W_{0}^{1, \Phi_{n}, \Psi_{n}}(\Omega), \\ +\infty, & \text { otherwise }\end{cases}
$$

Next theorem reveals which is the $\Gamma$-limit for the sequence $\left\{I_{n}\right\}$. Its proof follows the ideas from [5, Theorem 3.2] but we present it in detail for the convenience of the reader.

Theorem 4.1. Define $I_{\infty}: L^{1}(\Omega) \rightarrow[0, \infty]$ by

$$
I_{\infty}(u)= \begin{cases}0, & \text { if } u \in \mathcal{X} \text { and } \max \left\{\left\|\left|\nabla_{x} u\right|_{L}\right\|_{L^{\infty}(\Omega)},\left\|\left|\nabla_{y} u\right|_{M}\right\|_{L^{\infty}(\Omega)}\right\} \leq 1, \\ +\infty, & \text { otherwise. }\end{cases}
$$

where $\mathcal{X}=W^{1, \infty}(\Omega) \cap\left(\cap_{q \geq 1} W_{0}^{1, q}(\Omega)\right)$. Then $\Gamma\left(L^{1}(\Omega)\right)-\lim _{n \rightarrow \infty} I_{n}=I_{\infty}$.

A simple consequence of Theorem 4.1 which will be useful in establishing the conclusion of Theorem 3.1 is the following.

Corollary 4.2. For each integer $n \geq 1$ consider the functional $\bar{J}_{n}: L^{1}(\Omega) \rightarrow \overline{\mathbb{R}}$ defined by

$$
\bar{J}_{n}(u)= \begin{cases}J_{n}(u), & \text { if } u \in W_{0}^{1, \Phi_{n}, \Psi_{n}}(\Omega), \\ +\infty, & \text { otherwise }\end{cases}
$$

Define $\bar{J}_{\infty}: L^{1}(\Omega) \rightarrow \overline{\mathbb{R}}$ by

$\bar{J}_{\infty}(u)= \begin{cases}-\int_{\Omega} f u d \xi, & \text { if } u \in \mathcal{X} \text { and } \max \left\{\left\|\left|\nabla_{x} u\right|_{L}\right\|_{L^{\infty}(\Omega)},\left\|\left|\nabla_{y} u\right|_{M}\right\|_{L^{\infty}(\Omega)}\right\} \leq 1, \\ +\infty, & \text { otherwise. }\end{cases}$

where $\mathcal{X}=W^{1, \infty}(\Omega) \cap\left(\cap_{q>1} W_{0}^{1, q}(\Omega)\right)$. Then $\Gamma\left(L^{1}(\Omega)\right)-\lim _{n \rightarrow \infty} \bar{J}_{n}=\bar{J}_{\infty}$.

Proof of Theorem 4.1. We start by verifying the existence of a recovery sequence. If $I_{\infty}(u)=\infty$, the inequality clearly holds for any sequence $u_{n} \rightarrow u$ strongly in $L^{1}(\Omega)$. On the other hand, if $I_{\infty}(u)<+\infty$ we must have $I_{\infty}(u)=0$ and, as a result, $u \in \mathcal{X}=W^{1, \infty}(\Omega) \cap\left(\cap_{q \geq 1} W_{0}^{1, q}(\Omega)\right)$ and $\max \left\{\left\|\left|\nabla_{x} u\right|_{L}\right\|_{L^{\infty}(\Omega)},\left\|\left|\nabla_{y} u\right|_{M}\right\|_{L^{\infty}(\Omega)}\right\} \leq$ 1. For each $n \in \mathbb{N}$, let $u_{n}:=u$, and note that $u_{n} \in W_{0}^{1, \Phi_{n}, \Psi_{n}}(\Omega)$ and

$$
\begin{aligned}
\limsup _{n \rightarrow \infty} I_{n}\left(u_{n}\right) & =\limsup _{n \rightarrow \infty}\left(\int_{\Omega} \frac{\Phi_{n}\left(\left|\nabla_{x} u(\xi)\right|_{L}\right)}{\phi_{n}(1)} d \xi+\int_{\Omega} \frac{\Psi_{n}\left(\left|\nabla_{y} u(\xi)\right|_{M}\right)}{\psi_{n}(1)} d \xi\right) \\
& \leq \limsup _{n \rightarrow \infty}\left(\frac{|\Omega| \Phi_{n}(1)}{\phi_{n}(1)}+\frac{|\Omega| \Psi_{n}(1)}{\psi_{n}(1)}\right) \\
& \leq \limsup _{n \rightarrow \infty}\left(\frac{|\Omega|}{\phi_{n}^{-}}+\frac{|\Omega|}{\psi_{n}^{-}}\right)=0=I_{\infty}(u),
\end{aligned}
$$

where we have taken into account (1.3) and (2.10). This implies that the constant sequence $\left\{u_{n}\right\}=\{u\}$ is a recovery sequence for the $\Gamma$-limit.

To prove liminf-inequality from the definition of the $\Gamma$-convergence, we may assume, without loss of generality, that $u_{n} \in W_{0}^{1, \Phi_{n}, \Psi_{n}}(\Omega)$ and

$$
\liminf _{n \rightarrow \infty} I_{n}\left(u_{n}\right)=\lim _{n \rightarrow \infty} I_{n}\left(u_{n}\right)<\infty .
$$


Recall that $\Phi_{n}(t)$ dominates $t^{\phi_{n}^{-}}$near infinity for each $n \geq 1$, thus $L^{\Phi_{n}}(\Omega) \subset$ $L^{\phi_{n}^{-}}(\Omega)$ and hence, $\left|\nabla_{x} u_{n}\right|_{L} \in L^{\phi_{n}^{-}}(\Omega)$ (see [1, Lemma 8.12(b)]). Similarly, $\left|\nabla_{y} u_{n}\right|_{M} \in$ $L^{\psi_{n}^{-}}(\Omega)$, for each $n \geq 1$.

Let $\xi_{0} \in \Omega$ be a Lebesgue point for $\nabla u \in L^{1}\left(\Omega ; \mathbb{R}^{N}\right)$, namely

$$
\lim _{r \rightarrow 0^{+}} \frac{1}{\left|B_{r}\left(\xi_{0}\right)\right|} \int_{B_{r}\left(\xi_{0}\right)}\left|\nabla u(\eta)-\nabla u\left(\xi_{0}\right)\right|_{N} d \eta=0 .
$$

Since $\left|\nabla u(\eta)-\nabla u\left(\xi_{0}\right)\right|_{N}^{2}=\left|\nabla_{x} u(\eta)-\nabla_{x} u\left(\xi_{0}\right)\right|_{L}^{2}+\left|\nabla_{y} u(\eta)-\nabla_{y} u\left(\xi_{0}\right)\right|_{M}^{2}$ we deduce that

$\frac{1}{\left|B_{r}\left(\xi_{0}\right)\right|} \int_{B_{r}\left(\xi_{0}\right)}\left|\nabla_{x} u(\eta)-\nabla_{x} u\left(\xi_{0}\right)\right|_{L} d \eta \leq \frac{1}{\left|B_{r}\left(\xi_{0}\right)\right|} \int_{B_{r}\left(\xi_{0}\right)}\left|\nabla u(\eta)-\nabla u\left(\xi_{0}\right)\right|_{N} d \eta$,

and

$\frac{1}{\left|B_{r}\left(\xi_{0}\right)\right|} \int_{B_{r}\left(\xi_{0}\right)}\left|\nabla_{y} u(\eta)-\nabla_{y} u\left(\xi_{0}\right)\right|_{M} d \eta \leq \frac{1}{\left|B_{r}\left(\xi_{0}\right)\right|} \int_{B_{r}\left(\xi_{0}\right)}\left|\nabla u(\eta)-\nabla u\left(\xi_{0}\right)\right|_{N} d \eta$.

Consequently,

$$
\lim _{r \rightarrow 0^{+}} \frac{1}{\left|B_{r}\left(\xi_{0}\right)\right|} \int_{B_{r}\left(\xi_{0}\right)}\left|\nabla_{x} u(\eta)-\nabla_{x} u\left(\xi_{0}\right)\right|_{L} d \eta=0,
$$

and

$$
\lim _{r \rightarrow 0^{+}} \frac{1}{\left|B_{r}\left(\xi_{0}\right)\right|} \int_{B_{r}\left(\xi_{0}\right)}\left|\nabla_{y} u(\eta)-\nabla_{y} u\left(\xi_{0}\right)\right|_{M} d \eta=0,
$$

which shows that $\xi_{0}$ is a Lebesgue point for $\nabla_{x} u \in L^{1}\left(\Omega ; \mathbb{R}^{L}\right)$ and also for $\nabla_{y} u \in$ $L^{1}\left(\Omega ; \mathbb{R}^{M}\right)$. For any ball $B_{r}\left(\xi_{0}\right) \subset \Omega$, and $n \geq 1$ sufficiently large it holds, in view of Hölder's inequality, that

$$
\begin{aligned}
\int_{B_{r}\left(\xi_{0}\right)}\left|\nabla_{x} u_{n}(\eta)\right| d \eta & \leq\left\|\left|\nabla_{x} u_{n}\right|_{L}\right\|_{L^{\phi_{n}^{-}}}\left\|\chi_{B_{r}\left(\xi_{0}\right)}\right\|_{L^{\left(\phi_{n}^{-}\right)^{\prime}}} \\
& =\left\|\left|\nabla_{x} u_{n}\right|_{L}\right\|_{L^{\phi_{n}^{-}}}\left|B_{r}\left(\xi_{0}\right)\right|^{\frac{\phi_{n}^{-}-1}{\phi_{n}^{-}}}
\end{aligned}
$$

where $\left(\phi_{n}^{-}\right)^{\prime}:=\frac{\phi_{n}^{-}}{\phi_{n}^{-}-1}$.

Further, invoking [15, Lemma A.2] we have for all $n \geq 1, \rho>0$ and $t>0$ that

$$
\Phi_{n}(t)\left\{\begin{array}{ll}
\rho^{\phi_{n}^{+}} & \text {if } \rho \in(0,1] \\
\rho^{\phi_{n}^{-}} & \text {if } \rho \in(1, \infty)
\end{array} \leq \Phi_{n}(\rho t) \leq \Phi_{n}(t) \begin{cases}\rho^{\phi_{n}^{-}} & \text {if } \rho \in(0,1] \\
\rho^{\phi_{n}^{+}} & \text {if } \rho \in(1, \infty),\end{cases}\right.
$$

and

$$
\Psi_{n}(t)\left\{\begin{array}{ll}
\rho^{\psi_{n}^{+}} & \text {if } \rho \in(0,1] \\
\rho^{\psi_{n}^{-}} & \text {if } \rho \in(1, \infty)
\end{array} \leq \Psi_{n}(\rho t) \leq \Psi_{n}(t) \begin{cases}\rho^{\psi_{n}^{-}} & \text {if } \rho \in(0,1] \\
\rho^{\psi_{n}^{+}} & \text {if } \rho \in(1, \infty)\end{cases}\right.
$$

Consider the sets

$$
\Omega_{n}^{+}=\left\{\xi \in \Omega ;\left|\nabla_{x} u_{n}(\xi)\right|_{L} \geq 1\right\} \quad \text { and } \quad \Omega_{n}^{-}=\left\{\xi \in \Omega ;\left|\nabla_{x} u_{n}(\xi)\right|_{L}<1\right\} .
$$


Having in mind (1.4), (2.10) and (4.17), we can estimate

$$
\begin{aligned}
\int_{\Omega}\left|\nabla_{x} u_{n}(\xi)\right|_{L}^{\phi_{n}^{-}} d \xi & =\int_{\Omega_{n}^{-}}\left|\nabla_{x} u_{n}(x)\right|_{L}^{\phi_{n}^{-}} d \xi+\int_{\Omega_{n}^{+}}\left|\nabla_{x} u_{n}(\xi)\right|_{L}^{\phi_{n}^{-}} d \xi \\
& \leq|\Omega|+\int_{\Omega_{n}^{+}}\left|\nabla_{x} u_{n}(\xi)\right|_{L}^{\phi_{n}^{-}} d \xi \\
& \leq|\Omega|+\frac{\phi_{n}(1)}{\Phi_{n}(1)} \int_{\Omega} \frac{\Phi_{n}\left(\left|\nabla_{x} u_{n}(x)\right|_{L}\right)}{\phi_{n}(1)} d \xi \\
& \leq|\Omega|+\phi_{n}^{+} I_{n}\left(u_{n}\right) \leq|\Omega|+\beta \phi_{n}^{-} I_{n}\left(u_{n}\right) .
\end{aligned}
$$

Thus,

$$
\left\|\left|\nabla_{x} u_{n}\right|_{L}\right\|_{L^{\phi_{n}^{-}}} \leq\left[|\Omega|+\beta \phi_{n}^{-} I_{n}\left(u_{n}\right)\right]^{1 / \phi_{n}^{-}}
$$

which combined with (4.16) gives

$$
\int_{B_{r}\left(\xi_{0}\right)}\left|\nabla_{x} u_{n}(\eta)\right| d \eta \leq\left[|\Omega|+\beta \phi_{n}^{-} I_{n}\left(u_{n}\right)\right]^{1 / \phi_{n}^{-}}\left|B_{r}\left(\xi_{0}\right)\right|^{\frac{\phi_{n}^{-}-1}{\phi_{n}^{-}}} .
$$

Now we use (1.3) and (4.15) to infer that

$$
\limsup _{n \rightarrow \infty} \int_{B_{r}\left(\xi_{0}\right)}\left|\nabla_{x} u_{n}(\eta)\right|_{L} d \eta \leq\left|B_{r}\left(\xi_{0}\right)\right|
$$

and analogously,

$$
\limsup _{n \rightarrow \infty} \int_{B_{r}\left(\xi_{0}\right)}\left|\nabla_{y} u_{n}(\eta)\right|_{M} d \eta \leq\left|B_{r}\left(\xi_{0}\right)\right| .
$$

Let $q \geq 1$ be an arbitrary real number. By (1.3), $q<\phi_{n}^{-}$for sufficiently large $n \geq 1$. Applying Hölder's inequality we have

$$
\begin{aligned}
\int_{\Omega}\left|\nabla_{x} u_{n}(\xi)\right|_{L}^{q} d \xi & \leq\left(\int_{\Omega}\left|\nabla_{x} u_{n}(\xi)\right|_{L}^{\phi_{n}^{-}} d \xi\right)^{\frac{q}{\phi_{n}^{-}}}|\Omega|^{\frac{\phi_{n}^{-}-q}{\phi_{n}^{-}}} \\
& \leq\left(|\Omega|+\int_{\Omega} \frac{\Phi_{n}\left(\left|\nabla_{x} u_{n}(\xi)\right|_{L}\right)}{\Phi_{n}(1)} d \xi\right)^{\frac{q}{\phi_{n}^{-}}}|\Omega|^{\frac{\phi_{n}^{-}-q}{\phi_{n}^{-}}} \\
& \leq\left(|\Omega|+\beta \phi_{n}^{-} I_{n}\left(u_{n}\right)\right)^{\frac{q}{\phi_{n}^{-}}}|\Omega|^{\frac{\phi_{n}^{-}-q}{\phi_{n}^{-}}}
\end{aligned}
$$

Similarly, we obtain

$$
\int_{\Omega}\left|\nabla_{y} u_{n}(\xi)\right|_{M}^{q} d \xi \leq\left(|\Omega|+\beta \psi_{n}^{-} I_{n}\left(u_{n}\right)\right)^{\frac{q}{\psi_{n}^{-}}}|\Omega|^{\frac{\psi_{n}^{-}-q}{\psi_{n}^{-}}} .
$$

In particular this shows that the sequence $\left\{\nabla u_{n}\right\}$ is bounded in $L^{q}\left(\Omega ; \mathbb{R}^{N}\right)$ for any $q \geq 1$. The convergence $u_{n} \rightarrow u$ in $L^{1}(\Omega)$ guarantees that $\left\{u_{n}\right\}$ is bounded in $W_{0}^{1, q}(\Omega)$, and as a result we may extract a subsequence (not relabelled) such that $u_{n} \rightarrow u$ weakly in $W_{0}^{1, q}(\Omega)$. In particular, we find that $u \in \cap_{q \geq 1} W_{0}^{1, q}(\Omega)$. On the other hand, well-known lower semicontinuity results (see, e.g. [8, p. 61]) now give

$$
\int_{B_{r}\left(\xi_{0}\right)}\left|\nabla_{x} u(\eta)\right|_{L} d \eta \leq \liminf _{n \rightarrow \infty} \int_{B_{r}\left(\xi_{0}\right)}\left|\nabla_{x} u_{n}(\eta)\right|_{L} d \eta
$$

and

$$
\int_{B_{r}\left(\xi_{0}\right)}\left|\nabla_{y} u(\eta)\right|_{M} d \eta \leq \liminf _{n \rightarrow \infty} \int_{B_{r}\left(\xi_{0}\right)}\left|\nabla_{y} u_{n}(\eta)\right|_{M} d \eta
$$


Plugging (4.21) and (4.22) in the above inequalities implies that

$$
\frac{1}{\left|B_{r}\left(\xi_{0}\right)\right|} \int_{B_{r}\left(\xi_{0}\right)}\left|\nabla_{x} u(\eta)\right|_{L} d \eta \leq 1 \text { and } \frac{1}{\left|B_{r}\left(\xi_{0}\right)\right|} \int_{B_{r}\left(\xi_{0}\right)}\left|\nabla_{y} u(\eta)\right|_{M} d \eta \leq 1 .
$$

Observe that almost every $\xi_{0} \in \Omega$ is a Lebesgue point for $\nabla u$, and as shown above, a Lebesgue point for each $\nabla_{x} u$ and $\nabla_{y} u$ as well. If we pass to the limit $r \rightarrow 0^{+}$ in the above inequalities it yields $\left|\nabla_{x} u\left(\xi_{0}\right)\right|_{L} \leq 1$ and $\left|\nabla_{y} u\left(\xi_{0}\right)\right|_{M} \leq 1$ for a.e. $\xi_{0} \in \Omega$, or equivalently, $\max \left\{\left\|\left|\nabla_{x} u\right|_{L}\right\|_{L^{\infty}(\Omega)},\left\|\left|\nabla_{y} u\right|_{M}\right\|_{L^{\infty}(\Omega)}\right\} \leq 1$. Next, since $u \in \cap_{q>1} W_{0}^{1, q}(\Omega)$ we deduce that

$$
\int_{\Omega}|\nabla u|^{q} d x \geq \lambda_{1}(q) \int_{\Omega}|u|^{q} d x, \quad \forall q>1,
$$

where $\lambda_{1}(q)$ stands for the first eigenvalue of the $q$-Laplacian. In view of [21, Lemma 1.5] we know that $\lim _{q \rightarrow \infty}\left[\lambda_{1}(q)\right]^{1 / q}=\|\delta\|_{L^{\infty}(\Omega)}^{-1}$, where here $\delta$ is the distance function to the boundary of $\Omega$ with respect to the euclidian norm in $\mathbb{R}^{N}$. Thus, the above relations and the fact that $|\nabla u|_{N}^{2}=\left|\nabla_{x} u\right|_{L}^{2}+\left|\nabla_{y} u\right|_{M}^{2}$ now imply that

$$
\sqrt{2}>\left\||\nabla u|_{N}\right\|_{L^{\infty}(\Omega)} \geq \Lambda_{\infty}\|u\|_{L^{\infty}(\Omega)}
$$

and, thus, we deduce $u \in W^{1, \infty}(\Omega)$ and, consequently, $u \in \mathcal{X}$. It follows then that $I_{\infty}(u)=0$, which implies that liminf-inequality from the definition of $\Gamma$-convergence holds. This completes the proof of Theorem 4.1.

Finally, we conclude this section recalling the following well-known result which will be extremely helpful in obtaining Theorem 3.1. It can be found in [19, Corollary 6.1.1].

Proposition 4.1. Let $X$ be a topological space satisfying the first axiom of countability, and assume that the sequence $\left\{F_{n}\right\}$ of functionals $F_{n}: X \rightarrow \overline{\mathbb{R}}, \Gamma-$ converges to $F: X \rightarrow \overline{\mathbb{R}}$. Let $z_{n}$ be a minimizer for $F_{n}$. If $z_{n} \rightarrow z$ in $X$, then $z$ is a minimizer of $F$, and

$$
F(z)=\liminf _{n \rightarrow \infty} F_{n}\left(z_{n}\right) .
$$

\section{Proof of Theorem 3.1}

To accomplish the proof of our main result, we need first to establish the following proposition, which in the isotropic case and considering the $p$-Laplacian operator was obtained by Payne \& Philippin in [26].

Proposition 5.1. Assume that the hypotheses (1.1), (1.2), (1.3), and (1.4) hold, and for each integer $n \geq 1$ let $u_{n} \in W_{0}^{1, \Phi_{n}, \Psi_{n}}(\Omega)$ be the weak solution of the problem (1.5). Then

$$
\lim _{n \rightarrow \infty} \int_{\Omega} f(\xi) u_{n}(\xi) d \xi=\int_{\Omega} f(\xi) \delta_{1}(\xi) d \xi .
$$

The proof of Proposition 5.1 will require two auxiliary results, stated in Lemma 5.1 and Lemma 5.2 below.

Lemma 5.1. The sequence $\left\{\int_{\Omega} f(\xi) u_{n}(\xi) d \xi\right\}$ is bounded. 
Proof. For every integer $n \geq 1$ define

$$
m_{n}:=\min \left\{\phi_{n}^{-}, \psi_{n}^{-}\right\} .
$$

Then Hölder's inequality gives

$$
\int_{\Omega} f u_{n} d \xi \leq\|f\|_{L^{\infty}} \int_{\Omega} u_{n} d \xi \leq\|f\|_{L^{\infty}}\left(\int_{\Omega} u_{n}^{m_{n}} d \xi\right)^{1 / m_{n}}|\Omega|^{\left(m_{n}-1\right) / m_{n}} .
$$

From the above inequalities it is clear that it is enough to show that the sequence $\left\{\int_{\Omega} u_{n}(\xi) d \xi\right\}$ is bounded.

Denoting by $\lambda_{1}\left(m_{n}\right)$ the first eigenvalue of the $p$-Laplacian with $p=m_{n}$, given by

$$
\lambda_{1}\left(m_{n}\right):=\inf _{v \in C_{0}^{\infty}(\Omega) \backslash\{0\}} \frac{\int_{\Omega}|\nabla v|_{N}^{m_{n}} d \xi}{\int_{\Omega}|v|^{m_{n}} d \xi},
$$

we deduce that

$$
\left(\int_{\Omega} u_{n} d \xi\right)^{m_{n}} \leq|\Omega|^{m_{n}-1} \frac{\int_{\Omega}\left|\nabla u_{n}\right|_{N}^{m_{n}} d \xi}{\lambda_{1}\left(m_{n}\right)} .
$$

In view of (4.17) and (4.18) we find

$$
\Phi_{n}(t) \geq \Phi_{n}(1) t^{\phi_{n}^{-}} \quad \text { and } \quad \Psi_{n}(t) \geq \Psi_{n}(1) t^{\psi_{n}^{-}} \forall t>1,
$$

and hence

$$
t^{\phi_{n}^{-}} \leq 1+\frac{\Phi_{n}(t)}{\Phi_{n}(1)} \quad \text { and } \quad t^{\psi_{n}^{-}} \leq 1+\frac{\Psi_{n}(t)}{\Psi_{n}(1)} \quad \forall t>0 .
$$

In particular, taking $t$ as $\left|\nabla_{x} u_{n}(\xi)\right|_{L}$ and $\left|\nabla_{y} u_{n}(\xi)\right|_{M}$ these inequalities read as

$$
\begin{aligned}
& \left|\nabla_{x} u_{n}(\xi)\right|_{L}^{\phi_{n}^{-}} \leq 1+\frac{\Phi_{n}\left(\left|\nabla_{x} u_{n}(\xi)\right|_{L}\right)}{\Phi_{n}(1)} \\
& \left|\nabla_{y} u_{n}(\xi)\right|_{M}^{\psi_{n}^{-}} \leq 1+\frac{\Psi_{n}\left(\left|\nabla_{y} u_{n}(\xi)\right|_{M}\right)}{\Psi_{n}(1)}
\end{aligned}
$$

for all $\xi \in \Omega$ and for all integers $n \geq 1$.

On the other hand, elementary estimates and (5.24) yield

$$
\begin{aligned}
\left|\nabla u_{n}(\xi)\right|_{N}^{m_{n}} & =\left(\left|\nabla_{x} u_{n}(\xi)\right|_{L}^{2}+\left|\nabla_{y} u_{n}(\xi)\right|_{M}^{2}\right)^{m_{n} / 2} \\
& \leq 2^{\left(m_{n}-2\right) / 2}\left(\left|\nabla_{x} u_{n}(\xi)\right|_{L}^{m_{n}}+\left|\nabla_{y} u_{n}(\xi)\right|_{M}^{m_{n}}\right) \\
& \leq 2^{\left(m_{n}-2\right) / 2}\left(1+\left|\nabla_{x} u_{n}(\xi)\right|_{L}^{\phi_{n}^{-}}+\left|\nabla_{y} u_{n}(\xi)\right|_{M}^{\psi_{n}^{-}}\right) \\
& \leq 2^{\left(m_{n}-2\right) / 2}\left(3+\frac{\Phi_{n}\left(\left|\nabla_{x} u_{n}(\xi)\right|_{L}\right)}{\Phi_{n}(1)}+\frac{\Psi_{n}\left(\left|\nabla_{y} u_{n}(\xi)\right|_{M}\right)}{\Psi_{n}(1)}\right) .
\end{aligned}
$$


Using the above pieces of information and taking into account relations (2.10) and (3.14) we infer

$$
\begin{aligned}
& \left(\int_{\Omega} u_{n} d \xi\right)^{m_{n}} \\
& \leq \frac{|\Omega|^{m_{n}-1}}{\lambda_{1}\left(m_{n}\right)} 2^{\left(m_{n}-2\right) / 2}\left[3|\Omega|+\int_{\Omega} \frac{\Phi_{n}\left(\left|\nabla_{x} u_{n}(\xi)\right|_{L}\right)}{\Phi_{n}(1)} d \xi+\int_{\Omega} \frac{\Psi_{n}\left(\left|\nabla_{y} u_{n}(\xi)\right|_{M}\right)}{\Psi_{n}(1)} d \xi\right] \\
& \leq \frac{|\Omega|^{m_{n}-1}}{\lambda_{1}\left(m_{n}\right)} 2^{\left(m_{n}-2\right) / 2}\left[3|\Omega|+\frac{\phi_{n}(1)}{\Phi_{n}(1) \phi_{n}^{-}} \int_{\Omega} \frac{\phi_{n}\left(\left|\nabla_{x} u_{n}(\xi)\right|_{L}\right)\left|\nabla_{x} u_{n}(\xi)\right|_{L}}{\phi_{n}(1)} d \xi\right. \\
& \left.+\frac{\psi_{n}(1)}{\Psi_{n}(1) \psi_{n}^{-}} \int_{\Omega} \frac{\Psi_{n}\left(\left|\nabla_{y} u_{n}(\xi)\right|_{M}\right)\left|\nabla_{y} u_{n}(\xi)\right|_{M}}{\psi_{n}(1)} d \xi\right] \\
& \leq \frac{|\Omega|^{m_{n}-1}}{\lambda_{1}\left(m_{n}\right)} 2^{\left(m_{n}-2\right) / 2}\left[3|\Omega|+\frac{\phi_{n}^{+}}{\phi_{n}^{-}} \int_{\Omega} \frac{\phi_{n}\left(\left|\nabla_{x} u_{n}(\xi)\right|_{L}\right)\left|\nabla_{x} u_{n}(\xi)\right|_{L}}{\phi_{n}(1)} d \xi\right. \\
& \left.+\frac{\psi_{n}^{+}}{\psi_{n}^{-}} \int_{\Omega} \frac{\Psi_{n}\left(\left|\nabla_{y} u_{n}(\xi)\right|_{M}\right)\left|\nabla_{y} u_{n}(\xi)\right|_{M}}{\psi_{n}(1)} d \xi\right] \\
& \leq \frac{|\Omega|^{m_{n}-1}}{\lambda_{1}\left(m_{n}\right)} 2^{\left(m_{n}-2\right) / 2}\left[3|\Omega|+\beta \int_{\Omega} f u_{n} d \xi\right],
\end{aligned}
$$

and in consequence,

$$
\left(\int_{\Omega} u_{n} d \xi\right)^{m_{n}} \leq \frac{|\Omega|^{m_{n}-1}}{\lambda_{1}\left(m_{n}\right)} 2^{\left(m_{n}-2\right) / 2}\left[3|\Omega|+\beta\|f\|_{L^{\infty}} \int_{\Omega} u_{n} d \xi\right]
$$

The conclusion of the lemma now follows by contradiction. Indeed, if we assume that the sequence $\left\{\int_{\Omega} u_{n}(\xi) d \xi\right\}$ is unbounded, then there exists a subsequence, not relabelled, such that $\lim _{n \rightarrow \infty} \int_{\Omega} u_{n}(\xi) d \xi=+\infty$. Hence, for $n \geq 1$ sufficiently large, we have $3|\Omega| \leq \int_{\Omega} u_{n}(\xi) d \xi$. Dividing both sides of (5.25) by $\int_{\Omega} u_{n}(\xi) d \xi$ we find

$$
\begin{aligned}
\left(\int_{\Omega} u_{n} d \xi\right)^{m_{n}-1} & \leq \frac{|\Omega|^{m_{n}-1}}{\lambda_{1}\left(m_{n}\right)} 2^{\left(m_{n}-2\right) / 2}\left[\frac{3|\Omega|}{\int_{\Omega} u_{n} d \xi}+\beta\|f\|_{L^{\infty}}\right] \\
& \leq\left(1+\beta\|f\|_{\left.L^{\infty}\right)} 2^{\left(m_{n}-2\right) / 2} \frac{|\Omega|^{m_{n}-1}}{\lambda_{1}\left(m_{n}\right)}\right.
\end{aligned}
$$

or, equivalently, $\int_{\Omega} u_{n}(\xi) d \xi \leq\left(1+\beta\|f\|_{L^{\infty}}\right)^{1 /\left(m_{n}-1\right)} \frac{|\Omega| 2^{\left(m_{n}-2\right) /\left[2\left(m_{n}-1\right)\right]}}{\left\{\left[\lambda_{1}\left(m_{n}\right)\right]^{1 / m_{n}}\right\}^{m_{n} /\left(m_{n}-1\right)}}$. On the other hand, by $\left[21\right.$, Lemma 1.5] we know that $\lim _{n \rightarrow \infty}\left[\lambda_{1}\left(m_{n}\right)\right]^{1 / m_{n}}=$ $\|\delta\|_{L^{\infty}(\Omega)}^{-1}$, where here $\delta$ is the distance function to the boundary of $\Omega$ with respect to the euclidian norm in $\mathbb{R}^{N}$, which implies that the right-hand side in the last inequality above is bounded. This contradicts the fact that $\lim _{n \rightarrow \infty} \int_{\Omega} u_{n}(\xi) d \xi=\infty$. 
Lemma 5.2. There exists $u_{\infty} \in \mathcal{X}=W^{1, \infty}(\Omega) \cap\left(\cap_{q \geq 1} W_{0}^{1, q}(\Omega)\right)$ with

$$
\max \left\{\left\|\left|\nabla_{x} u_{\infty}\right|_{L}\right\|_{L^{\infty}(\Omega)},\left\|\left|\nabla_{y} u_{\infty}\right|_{M}\right\|_{L^{\infty}(\Omega)}\right\} \leq 1,
$$

and a subsequence of $\left\{u_{n}\right\}$ (not relabeled) such that $u_{n} \rightarrow u_{\infty}$ uniformly in $\Omega$.

Proof. Let $q \geq N$ be an arbitrary real number. By (1.3), $q<\phi_{n}^{-}$and $q<\psi_{n}^{-}$for sufficiently large integers $n \geq 1$. Using Hölder's inequality, (5.24), recalling the fact that $\left\langle J_{n}^{\prime}\left(u_{n}\right), u_{n}\right\rangle=0$, and taking into account (1.4) and (2.10), we deduce that

$$
\begin{aligned}
\int_{\Omega}\left|\nabla_{x} u_{n}(\xi)\right|_{L}^{q} d \xi & \leq\left[\int_{\Omega}\left|\nabla_{x} u_{n}(\xi)\right|_{L}^{\phi_{n}^{-}} d \xi\right]^{\frac{q}{\phi_{n}^{-}}}|\Omega|^{\frac{\phi_{n}^{-}-q}{\phi_{n}^{-}}} \\
& \leq\left[|\Omega|+\int_{\Omega} \frac{\Phi_{n}\left(\left|\nabla_{x} u_{n}(\xi)\right|_{L}\right)}{\Phi_{n}(1)} d \xi\right]^{\frac{q}{\phi_{n}^{-}}}|\Omega|^{\frac{\phi_{n}^{-}-q}{\phi_{n}^{-}}} \\
& \leq\left[|\Omega|+\frac{\phi_{n}(1)}{\Phi_{n}(1) \phi_{n}^{-}} \int_{\Omega} \frac{\phi_{n}\left(\left|\nabla_{x} u_{n}(\xi)\right|_{L}\right)\left|\nabla_{x} u_{n}(\xi)\right|_{L}}{\phi_{n}(1)} d \xi\right]^{\frac{q}{\phi_{n}^{-}}}|\Omega|^{\frac{\phi_{n}^{-}-q}{\phi_{n}^{-}}} \\
& \leq\left[|\Omega|+\frac{\phi_{n}^{+}}{\phi_{n}^{-}} \int_{\Omega} f(\xi) u_{n}(\xi) d \xi\right]^{\frac{q}{\phi_{n}^{-}}}|\Omega|^{\frac{\phi_{n}^{-}-q}{\phi_{n}^{-}}} \\
& \leq\left[|\Omega|+\beta \int_{\Omega} f(\xi) u_{n}(\xi) d \xi\right]^{\frac{q}{\phi_{n}^{-}}}|\Omega|^{\frac{\phi_{n}^{-}-q}{\phi_{n}^{-}}} .
\end{aligned}
$$

Similarly, we can obtain

$$
\int_{\Omega}\left|\nabla_{y} u_{n}(\xi)\right|_{M}^{q} d \xi \leq\left[|\Omega|+\beta \int_{\Omega} f(\xi) u_{n}(\xi) d \xi\right]^{\frac{q}{\psi_{n}^{-}}}|\Omega|^{\frac{\psi_{n}^{-}-q}{\psi_{n}^{-}}} .
$$

By Lemma 5.1, there exists a positive constant $M$ such that $\int_{\Omega} f(\xi) u_{n}(\xi) d \xi \leq M$ for all integers $n \geq 1$ sufficiently large. Thus, for all such $n \geq 1$ we must have

$$
\left\|\left|\nabla_{x} u_{n}\right|_{L}\right\|_{L^{q}(\Omega)} \leq(|\Omega|+\beta M)^{1 / \phi_{n}^{-}}|\Omega|^{\left(\phi_{n}^{-}-q\right) /\left(q \phi_{n}^{-}\right)},
$$

and

$$
\left\|\left|\nabla_{y} u_{n}\right|_{M}\right\|_{L^{q}(\Omega)} \leq(|\Omega|+\beta M)^{1 / \psi_{n}^{-}}|\Omega|^{\left(\psi_{n}^{-}-q\right) /\left(q \psi_{n}^{-}\right)} .
$$

This assures that the sequence $\left\{\nabla u_{n}\right\}$ is bounded in $L^{q}\left(\Omega ; \mathbb{R}^{N}\right)$ for any $q \geq N$. Hence, there exists a subsequence $\left\{u_{n_{i}}\right\}$ of $\left\{u_{n}\right\}$ and $u_{\infty} \in W_{0}^{1, q}(\Omega)$, such that $u_{n_{i}} \rightarrow u_{\infty}$ weakly in $W_{0}^{1, q}(\Omega)$ and $u_{n_{i}} \rightarrow u_{\infty}$ strongly in $L^{q}(\Omega)$. However, since $q \geq$ $N$ was arbitrary, the compactness of the embedding of $W_{0}^{1, q}(\Omega)$ into $C^{0, \alpha}(\Omega)(0<$ $\alpha<1$ ) for $q>N$ (one can choose, e.g., $\alpha=1-N / q$ ), allows us to conclude that indeed $u_{n_{i}} \rightarrow u_{\infty}$ uniformly in $\Omega$. Finally, Proposition 4.1 (with $X=L^{1}(\Omega)$, $F_{n}=\bar{J}_{n}, F_{\infty}=\bar{J}_{\infty}, z_{n}=u_{n}$ ) and Corollary 4.2 ensure that $u_{\infty}$ is a minimizer for $\bar{J}_{\infty}$ which, in particular, means that $u_{\infty} \in \mathcal{X}$ and

$$
\max \left\{\left\|\left|\nabla_{x} u_{\infty}\right|_{L}\right\|_{L^{\infty}(\Omega)},\left\|\left|\nabla_{y} u_{\infty}\right|_{M}\right\|_{L^{\infty}(\Omega)}\right\} \leq 1 .
$$

Lemma 5.2 is proved.

Proof of Proposition 5.1. Fix an arbitrary subsequence of $\left\{u_{n}\right\}$, still denoted by $\left\{u_{n}\right\}$. Similar arguments as those used in the proof of Lemma 5.2 can be 
considered to show that this subsequence contains, in its turn, a subsequence, say $\left\{u_{n_{k}}\right\}$, which converges uniformly in $\Omega$ to a certain limit $u_{\infty} \in \mathcal{X}$ with

$$
\max \left\{\left\|\left|\nabla_{x} u_{\infty}\right|_{L}\right\|_{L^{\infty}(\Omega)},\left\|\left|\nabla_{y} u_{\infty}\right|_{M}\right\|_{L^{\infty}(\Omega)}\right\} \leq 1 .
$$

In order to get the conclusion of Proposition 5.1 it is enough to establish that $\lim _{k \rightarrow \infty} \int_{\Omega} f u_{n_{k}} d \xi=\int_{\Omega} f \delta_{1} d \xi$. In other words, we will show that the limit of all possible subsequences of $\left\{\int_{\Omega} f u_{n} d \xi\right\}$ is $\int_{\Omega} f \delta_{1} d \xi$ and in accordance, the limit of the full sequence should also be $\int_{\Omega} f \delta_{1} d \xi$.

In the sequel, for simplicity, we will write $u_{n}$ instead of $u_{n_{k}}$.

Since $\delta_{1} \in \mathcal{X} \subset W_{0}^{1, \Phi_{n}, \Psi_{n}}(\Omega), \max \left\{\left\|\left|\nabla_{x} \delta_{1}\right|_{L}\right\|_{L^{\infty}},\left\|\left|\nabla_{y} \delta_{1}\right|_{M}\right\|_{L^{\infty}}\right\}=1$ (see, e.g. [3, p.37, Section 3]) and $u_{n}$ is a minimizer of $J_{n}$ in $W_{0}^{1, \Phi_{n}, \Psi_{n}}(\Omega)$, for each integer $n \geq 1$ it holds that

$$
J_{n}\left(u_{n}\right) \leq J_{n}\left(\delta_{1}\right)=\int_{\Omega} \frac{\Phi_{n}\left(\left|\nabla_{x} \delta_{1}\right|_{L}\right)}{\phi_{n}(1)} d \xi+\int_{\Omega} \frac{\Psi_{n}\left(\left|\nabla_{y} \delta_{1}\right|_{M}\right)}{\psi_{n}(1)} d \xi-\int_{\Omega} f \delta_{1} d \xi,
$$

or,

$$
\begin{aligned}
\int_{\Omega} f \delta_{1} d \xi & \leq \frac{|\Omega| \Phi_{n}(1)}{\phi_{n}(1)}+\frac{|\Omega| \Psi_{n}(1)}{\psi_{n}(1)}+\int_{\Omega} f u_{n} d \xi \\
& \leq \frac{|\Omega|}{\phi_{n}^{-}}+\frac{|\Omega|}{\psi_{n}^{-}}+\int_{\Omega} f u_{n} d \xi, \quad \forall n \geq 1 .
\end{aligned}
$$

Consequently, we find

$$
\int_{\Omega} f \delta_{1} d \xi \leq \lim _{n \rightarrow \infty} \int_{\Omega} f u_{n} d \xi=\int_{\Omega} f u_{\infty} d \xi
$$

Next, we observe that for each $\xi=(x, y) \in \Omega$ and $\eta=(a, b) \in \partial \Omega$ such that ]$\xi-\eta\left[1=\delta_{1}(\xi)\right.$, we have

$$
\begin{aligned}
u_{\infty}(\xi) & =u_{\infty}(\xi)-u_{\infty}(\eta) \\
& \leq|x-a|_{L}\left\|\left.\left|\nabla_{x} u_{\infty}\right|\right|_{L}\right\|_{L^{\infty}}+|y-b|_{M}\left\|\left|\nabla_{y} u_{\infty}\right|_{M}\right\|_{L^{\infty}} \\
& \leq \delta_{1}(\xi) .
\end{aligned}
$$

Multiplying by $f$ and integrating over $\Omega$ we get

$$
\lim _{n \rightarrow \infty} \int_{\Omega} f u_{n} d \xi=\int_{\Omega} f u_{\infty} d \xi \leq \int_{\Omega} f \delta_{1} d \xi
$$

Recalling (5.28), we deduce that $\lim _{n \rightarrow \infty} \int_{\Omega} f u_{n} d \xi=\int_{\Omega} f \delta_{1} d \xi$, which concludes the proof of Proposition 5.1.

We are now ready to give the proof of Theorem 3.1.

Proof of Theorem 3.1. As in Proposition 5.1 we fix an arbitrary subsequence of the solutions $\left\{u_{n}\right\}$ (not relabeled). Again as in Lemma 5.2 we can admit that $\left\{u_{n}\right\}$ converges uniformly to a certain limit $u_{\infty} \in \mathcal{X}$ with

$$
\max \left\{\left\|\left|\nabla_{x} u_{\infty}\right|_{L}\right\|_{L^{\infty}(\Omega)},\left\|\left|\nabla_{y} u_{\infty}\right|_{M}\right\|_{L^{\infty}(\Omega)}\right\} \leq 1 .
$$

Hence, it just remains to see that $u_{\infty}=\delta_{1}$. Notice that, since $\left\{u_{n}\right\}$ is arbitrary, this means that $\delta_{1}$ is indeed the limit of the full sequence $\left\{u_{n}\right\}$. Recall that by (5.29) we have $u_{\infty}(\xi) \leq \delta_{1}(\xi)$, for each $\xi \in \Omega$. Further, since $u_{n}(\xi) \geq 0$ for a.e. $\xi \in \Omega$ and 
for every integer $n \geq 1$, we deduce that $u_{\infty}(\xi) \geq 0$ for a.e. $\xi \in \Omega$. Finally, applying Proposition 5.1 and taking into account the fact that $u_{n} \rightarrow u_{\infty}$ uniformly in $\Omega$, we find that

$$
\int_{\Omega} f \delta_{1} d \xi=\lim _{n \rightarrow \infty} \int_{\Omega} f u_{n} d \xi=\int_{\Omega} f u_{\infty} d \xi
$$

Recalling the continuity of $f, \delta_{1}$ and $u_{\infty}$, the last equalities yield $u_{\infty}=\delta$. The proof of Theorem 3.1 is complete.

\section{TheOREM 3.1 REVISITED UNDER THE POINT OF VIEW OF VISCOSITY SOLUTIONS}

We devote this section to show our main result under a completely different perspective: the identification of the limit equation as $n \rightarrow \infty$ in (1.5), which has to be understood in viscosity sense, see Definitions 6.1, 6.2 and 6.3 below. As we will see the anisotropic distance also fulfils the limit equation, and then the identification $u_{\infty}=\delta_{1}$ is a consequence of the uniqueness of solutions.

Furthermore, these arguments lead as well that the limit $u_{\infty}$ maximizes the functional $F(v)=\int_{\Omega} f v$ among certain set of functions. With this result and the positivity of $f$, the fact that $u_{\infty}=\delta_{1}$ follows straightforward.

Let us write in detail these ideas. We start by specifying the notion of solutions we are considering along this section. We refer to [10] for the definition and main properties of viscosity solutions to problems of the type

$$
\begin{cases}H_{n}\left(u_{n}, \nabla u_{n}, D^{2} u_{n}\right)=0, & \text { in } \Omega, \\ u_{n}=0 & \text { on } \partial \Omega,\end{cases}
$$

where $D^{2} u_{n}$ stands for the Hessian matrix of $u_{n}$.

Definition 6.1. An upper semicontinuous function $u$ defined in $\Omega$ is a viscosity subsolution of (6.30) if, $\left.u\right|_{\partial \Omega} \leq 0$ and, whenever $x_{0} \in \Omega$ and $\psi \in C^{2}(\Omega)$ are such that $u\left(x_{0}\right)=\psi\left(x_{0}\right)$ and $u(x)<\psi(x)$, if $x \neq x_{0}$, then

$$
H_{n}\left(u_{n}, \nabla u_{n}, D^{2} u_{n}\right) \leq 0 \text {. }
$$

Definition 6.2. A lower semicontinuous function $u$ defined in $\Omega$ is a viscosity supersolution of (6.30) if, $\left.u\right|_{\partial \Omega} \geq 0$ and, whenever $x_{0} \in \Omega$ and $\phi \in C^{2}(\Omega)$ are such that $u\left(x_{0}\right)=\phi\left(x_{0}\right)$ and $u(x)>\phi(x)$, if $x \neq x_{0}$, then

$$
H_{n}\left(u_{n}, \nabla u_{n}, D^{2} u_{n}\right) \geq 0 .
$$

Definition 6.3. A continuos function that is both supersolution and subsolution to (6.30) is a viscosity solution to (6.30).

Note that in both of the above definitions the strict inequality can be relaxed, since the second condition is required just in a neigbourhood of $x_{0}$. We refer to [10] for further details about general theory of viscosity solutions, and [4, 20, 22] for viscosity solutions related to the $\infty$-Laplacian and the $p$-Laplacian operators. Regarding viscosity solutions to anisotropic operators we refer for instance to [3, 14, 16, 27, 28]. More recently, in [6] the authors determine the limit equation corresponding to the isotropic case of (1.5), which turns out to be the same limit as in $[4,29]$. In view of this, it would be reasonable to think that the limit equation in our case coincide with the limit found in [14], and this is indeed what occurs, see Proposition 6.1 below. 
If we admit that the solutions $u_{n}$ to (1.5) are smooth enough so that we can take derivatives in the PDE, our problem could be rewritten as follows

$$
\begin{aligned}
& -\frac{\phi_{n}\left(\left|\nabla_{x} u_{n}\right|_{L}\right)}{\phi_{n}(1)\left|\nabla_{x} u_{n}\right|_{L}} \Delta_{x} u_{n}-\frac{\left|\nabla_{x} u_{n}\right|_{L} \phi_{n}^{\prime}\left(\left|\nabla_{x} u_{n}\right|_{L}\right)-\phi_{n}\left(\left|\nabla_{x} u_{n}\right|_{L}\right)}{\phi_{n}(1)\left|\nabla_{x} u_{n}\right|_{L}^{3}} \Delta_{\infty, x} u_{n} \\
& \quad-\frac{\psi_{n}\left(\left|\nabla_{y} u_{n}\right|_{M}\right)}{\psi_{n}(1)\left|\nabla_{y} u_{n}\right|_{M}} \Delta_{y} u_{n}-\frac{\left|\nabla_{y} u_{n}\right|_{M} \psi_{n}^{\prime}\left(\left|\nabla_{y} u_{n}\right|_{M}\right)-\psi_{n}\left(\left|\nabla_{y} u_{n}\right|_{M}\right)}{\psi_{n}(1)\left|\nabla_{y} u_{n}\right|_{M}^{3}} \Delta_{\infty, y} u_{n}=f .
\end{aligned}
$$

Here $\Delta_{x} v=\operatorname{div}_{x}\left(\nabla_{x} v\right), \Delta_{y} v=\operatorname{div}_{y}\left(\nabla_{y} v\right), \Delta_{\infty, x} v=\nabla_{x} v D_{x}^{2} v\left(\nabla_{x} v\right)^{t}$ and $\Delta_{\infty, y} v=$ $\nabla_{y} v D_{y}^{2} v\left(\nabla_{y} v\right)^{t}$ are the Laplacian and the infinity Laplacian in $x$ variables and in $y$ variables, respectively.

Let $z \in \mathbb{R}^{N}$ and

$$
w_{1}=\left(z_{1}, \ldots, z_{L}\right), \quad \text { and } \quad w_{2}=\left(z_{L+1}, \ldots, z_{N}\right),
$$

stand for the first $L$ components and for the last $M$ components of $z$. Also for $S \in \mathbb{S}^{N}$ we will call

$$
S_{1}=\left(s_{i j}\right)_{1 \leq i, j \leq L}
$$

the first $L \times L$ minor of the matrix $S$ and

$$
S_{2}=\left(s_{i j}\right)_{L+1 \leq i, j \leq N}
$$

the last $M \times M$ minor of $S$.

According to the notation adopted in (6.30) if we let

$$
\begin{aligned}
H_{n}(w, S):= & -\frac{\phi_{n}\left(\left|w_{1}\right|_{L}\right)}{\phi_{n}(1)\left|w_{1}\right|_{L}} \operatorname{trace}\left(S_{1}\right)-\frac{\left|w_{1}\right|_{L} \phi_{n}^{\prime}\left(\left|w_{1}\right|_{L}\right)-\phi_{n}\left(\left|w_{1}\right|_{L}\right)}{\phi_{n}(1)\left|w_{1}\right|_{L}^{3}}\left\langle S_{1} w_{1}, w_{1}\right\rangle \\
& -\frac{\psi_{n}\left(\left|w_{2}\right|_{M}\right)}{\psi_{n}(1)\left|w_{2}\right|_{M}} \operatorname{trace}\left(S_{2}\right)-\frac{\left|w_{2}\right|_{M} \psi_{n}^{\prime}\left(\left|w_{2}\right|_{M}\right)-\psi_{n}\left(\left|w_{2}\right|_{M}\right)}{\psi_{n}(1)\left|w_{2}\right|_{M}^{3}}\left\langle S_{2} w_{2}, w_{2}\right\rangle
\end{aligned}
$$

then we can write

$$
H_{n}\left(\nabla u_{n}, D^{2}\left(u_{n}\right)\right)=f .
$$

It is standard to show that if $u_{n}$ is a continuous weak solution to (1.5) then it is a viscosity solution to (6.31), see for example Lemma 1.8 in [21].

Notice that with the only use of the equation (1.5) all of the results stated in Lemma 5.2 can be obtained, since in (5.26) and (5.27) we can pass to the limit as $\phi_{n}^{-}$and $\psi_{n}^{-}$go to $\infty$, and then as $q \rightarrow \infty$ to prove that

$$
\max \left\{\left\|\left|\nabla_{x} u_{\infty}\right|_{L}\right\|_{L^{\infty}(\Omega)},\left\|\left|\nabla_{y} u_{\infty}\right|_{M}\right\|_{L^{\infty}(\Omega)}\right\} \leq 1,
$$

being $u_{\infty}$ the uniform limit in $\Omega$ of some subsequence of $\left\{u_{n}\right\}$. Furthermore, for $n$ sufficiently large, $u_{n}$ are continuous weak solutions to (1.5), hence viscosity solutions to $(6.30)$. In order to identify the limit equation for such $u_{\infty}$, we need to show certain properties satisfied by this limit summarized in the next lemma. These results are inspired from some ideas presented in [14], but we include the details of proof for convenience of the reader.

Lemma 6.1. Let $u_{\infty}$ be the limit obtained in Lemma 5.2. Then, $u_{\infty}$ maximizes the functional

$$
F(v):=\int_{\Omega} f v d \xi
$$

in the set

$$
K=\left\{v \in \mathcal{X}: \max \left\{\left\|\left|\nabla_{x} u\right|_{L}\right\|_{L^{\infty}(\Omega)},\left\|\left|\nabla_{y} u\right|_{M}\right\|_{L^{\infty}(\Omega)}\right\} \leq 1\right\},
$$


where $\mathcal{X}=W^{1, \infty}(\Omega) \cap\left(\cap_{q \geq 1} W_{0}^{1, q}(\Omega)\right)$, namely,

$$
F\left(u_{\infty}\right)=\max _{v \in K} F(v) .
$$

Furthermore, $u_{\infty}$ also maximizes (6.32) if $F$ is integrated in any subdomain of $\Omega$.

Finally, if $D \subset \Omega$ is a convex set, then for every $\xi \in D$, we can express $u_{\infty}$ as

$$
u_{\infty}(\xi)=\inf _{\theta \in A(\xi)}\left\{u_{\infty}(\theta)+\right] \xi-\theta[1\},
$$

where $A(\xi)=\left\{\theta \in \partial D\right.$ such that $\left.\delta_{1}(\xi, \partial D):=\inf _{\eta \in \partial D}\right] \xi, \eta\left[{ }_{1}=\right] \xi, \theta\left[{ }_{1}\right\}$.

Remark 6.1. Note that the property of $u_{\infty}$ given by Lemma 6.1, namely $F\left(u_{\infty}\right)=$ $\max _{v \in K} F(v)$, can be related with the $\Gamma$-convergence results from Section 4 .

Proof. Observe that $u_{n}$ are minimizers of the Euler-Lagrange functional given by (3.13). In particular, for any $v \in K$

$-\int_{\Omega} f u_{n} d \xi \leq J_{n}\left(u_{n}\right) \leq \frac{|\Omega| \Phi_{n}(1)}{\phi_{n}(1)}+\frac{|\Omega| \Psi_{n}(1)}{\psi_{n}(1)}-\int_{\Omega} f v d \xi \leq \frac{|\Omega|}{\phi_{n}^{-}}+\frac{|\Omega|}{\psi_{n}^{-}}+\int_{\Omega} f v d \xi$.

If we pass to the limit as $n \rightarrow \infty$ in the previous chain of inequalities, using (1.3) and the convergence $u_{n} \rightarrow u_{\infty}$ uniformly in $\Omega$, we get

$$
\int_{\Omega} f u_{\infty} d \xi \geq \int_{\Omega} f v d \xi, \text { for any } v \in K
$$

Let us now define the set

$\widetilde{K}=\left\{v \in W^{1, \infty}(D): \max \left\{\left\|\left|\nabla_{x} v\right|_{L}\right\|_{L^{\infty}(D)},\left\|\left|\nabla_{y} v\right|_{M}\right\|_{L^{\infty}(D)}\right\} \leq 1,\left.v\right|_{\partial D}=\left.u_{\infty}\right|_{\partial D}\right\}$, with $D \subseteq \Omega$ being an open and smooth subset. Since $\left.u_{\infty}\right|_{D} \in \widetilde{K}$, it follows that

$$
\max _{v \in \widetilde{K}} \int_{D} f v d \xi \geq \int_{D} f u_{\infty} d \xi .
$$

By contradiction, suppose that there exists $v^{*} \in \widetilde{K}$ such that $\int_{D} f v^{*} d \xi>\int_{D} f u_{\infty} d \xi$. Then we can construct $u^{*} \in K$ as follows

$$
u^{*}= \begin{cases}v^{*} & \text { in } \mathrm{D} \\ u_{\infty} & \text { in } \Omega \backslash \mathrm{D} .\end{cases}
$$

Clearly $\int_{\Omega} f u^{*} d \xi>\int_{\Omega} f u_{\infty} d \xi$, which contradicts the previous assertion. Actually, the above arguments show that

$$
\int_{D} f u_{\infty} d \xi=\max _{v \in \widetilde{K}} \int_{D} f v d \xi
$$

provided that $D \subseteq \Omega$ is an open and smooth subset.

Finally, assume that $D \subset \Omega$ is a convex subset. Let $\xi \in D$ be fixed. Since $u_{\infty} \in K$ we have $\left.u_{\infty}(\xi) \leq u_{\infty}(\theta)+\right] \xi-\theta\left[{ }_{1}\right.$, for every $\theta \in \partial D$ such that $\delta_{1}(\xi, \partial D)$ is attained, i.e, for every $\theta \in A(\xi)$. In particular, we have

$$
u_{\infty}(\xi) \leq \inf _{\eta \in A(\xi)}\left\{u_{\infty}(\eta)+\right] \xi-\eta[1\} .
$$

We construct $v \in \tilde{K}$ as follows

$$
v(\xi)=\inf _{\eta \in A(\xi)}\left\{u_{\infty}(\eta)+\right] \xi-\eta[1\} .
$$

Note that $v$ is a continuous function over $D$ which satisfies

$$
\max \left\{\left\|\left|\nabla_{x} v\right|_{L}\right\|_{L^{\infty}(D)},\left\|\left|\nabla_{y} v\right|_{M}\right\|_{L^{\infty}(D)}\right\} \leq 1,
$$


and $\left.v\right|_{\partial D} \equiv u_{\infty}$. Then, by (6.33) we have

$$
\int_{D} f v d \xi \leq \int_{D} f u_{\infty} d \xi
$$

On the other hand, (6.34) yields that $u_{\infty} \leq v$ in $D$. Integrating this inequality over $D$ and taking into account relation (6.35) we deduce that

$$
\int_{D} f v d \xi=\int_{D} f u_{\infty} d \xi
$$

Combining this equality with that fact that $v$ and $u_{\infty}$ are continuous functions satisfying $u_{\infty} \leq v$ in $D$ we deduce that $u_{\infty}=v$ in $D$. The proof of Lemma 6.1 is now complete.

We are ready now to identify the limit equation for $u_{\infty}$.

Theorem 6.1. Let $u_{\infty}$ be the limit obtained in Lemma 5.2. Then $u_{\infty}$ is the unique viscosity solution of the following equation

$$
\begin{cases}\max \left\{\left|\nabla_{x} u_{\infty}\right|_{L},\left|\nabla_{y} u_{\infty}\right|_{M}\right\}=1 & \text { in } \Omega \\ u_{\infty}=0 & \text { on } \partial \Omega .\end{cases}
$$

Proof. The uniqueness of the viscosity solutions for equation (6.36) is a simple consequence of [17, Theorem 1]. Thus, it remains to show that $u_{\infty}$ is a solution of (6.36). In order to do that we first observe that $u_{\infty}=0$ on $\partial \Omega$, since $u_{n}=0$ on $\partial \Omega$ for each positive integer $n$. Next, we will show that $u_{\infty}$ is both a viscosity supersolution and a viscosity subsolution of the above quoted equation.

Let us consider a point $\xi_{0} \in \Omega$. To prove that $u_{\infty}$ is a viscosity supersolution, let $\varphi$ be a function in $C^{2}(\Omega)$ such that $u_{\infty}\left(\xi_{0}\right)=\varphi\left(\xi_{0}\right)$ and $u_{\infty}-\varphi$ has a local minimum at $\xi_{0}$. Since $u_{n} \rightarrow u_{\infty}$ uniformly, there exists a sequence $\xi_{n} \rightarrow \xi_{0}$ such that $u_{n}-\varphi$ has a local minimum at $\xi_{n}$, for any positive integer $n$.

Recall that $u_{n}$ are viscosity solutions to (6.31) and in particular

$$
\begin{aligned}
& -\frac{\phi_{n}\left(\left|\nabla_{x} \varphi\left(\xi_{n}\right)\right|_{L}\right)}{\phi_{n}(1)\left|\nabla_{x} \varphi\left(\xi_{n}\right)\right|_{L}} \Delta_{x} \varphi\left(\xi_{n}\right)-\frac{\psi_{n}\left(\left|\nabla_{y} \varphi\left(\xi_{n}\right)\right|_{M}\right)}{\psi_{n}(1)\left|\nabla_{y} \varphi\left(\xi_{n}\right)\right|_{M}} \Delta_{y} \varphi\left(\xi_{n}\right) \\
& -\frac{\left|\nabla_{x} \varphi\left(\xi_{n}\right)\right|_{L} \phi_{n}^{\prime}\left(\left|\nabla_{x} \varphi\left(\xi_{n}\right)\right|_{L}\right)-\phi_{n}\left(\left|\nabla_{x} \varphi\left(\xi_{n}\right)\right|_{L}\right)}{\phi_{n}(1)\left|\nabla_{x} \varphi\left(\xi_{n}\right)\right|_{L}^{3}} \Delta_{\infty, x} \varphi\left(\xi_{n}\right) \\
& \left.\quad-\frac{\left|\nabla_{y} \varphi\left(\xi_{n}\right)\right|_{M} \psi_{n}^{\prime}\left(\left|\nabla_{y} \varphi\left(\xi_{n}\right)\right|_{M}\right)-\psi_{n}\left(\left|\nabla_{y} \varphi\left(\xi_{n}\right)\right|_{M}\right)}{\psi_{n}(1)\left|\nabla_{y} \varphi\left(\xi_{n}\right)\right|_{M}^{3}} \Delta_{\infty, y} \varphi\left(\xi_{n}\right)\right\rangle \geq f\left(\xi_{n}\right) .
\end{aligned}
$$

Note that the estimates from [25, Lemma 2.1]

$$
\begin{aligned}
& \min \left\{s^{\phi_{n}^{-}}, s^{\phi_{n}^{+}}\right\} \phi_{n}(t) \leq \phi_{n}(s t) \leq \max \left\{s^{\phi_{n}^{-}}, s^{\phi_{n}^{+}}\right\} \phi_{n}(t), \\
& \min \left\{s^{\psi_{n}^{-}}, s^{\psi_{n}^{+}}\right\} \psi_{n}(t) \leq \psi_{n}(s t) \leq \max \left\{s^{\psi_{n}^{-}}, s^{\psi_{n}^{+}}\right\} \psi_{n}(t),
\end{aligned}
$$

for all $t, s \geq 0$ together with (1.3) ensure that the functions $b_{n}, \tilde{b}_{n}:[0, \infty) \rightarrow \mathbb{R}$ defined by $b_{n}(t):=\frac{t \phi_{n}^{\prime}(t)-\phi_{n}(t)}{t^{3}}$ and reciprocally $\tilde{b}_{n}(t):=\frac{t \psi_{n}^{\prime}(t)-\psi_{n}(t)}{t^{3}}$ if $t>0$, $b_{n}(0):=0$ and $\tilde{b}_{n}(0):=0$ are continuous. In addition, the functions $a_{n}, \tilde{a}_{n}$ : $[0, \infty) \rightarrow \mathbb{R}$ given by $a_{n}(t):=\frac{\phi_{n}(t)}{t}$ and $\tilde{a}_{n}(t):=\frac{\psi_{n}(t)}{t}$ if $t>0, a_{n}(0):=0$ and $\tilde{a}_{n}(0):=0$ are of class $C^{1}$ on $(0, \infty)$. 
Assume by contradiction that

$$
\max \left\{\left|\nabla_{x} \varphi\left(\xi_{0}\right)\right|_{L},\left|\nabla_{y} \varphi\left(\xi_{0}\right)\right|_{M}\right\}<1 .
$$

It follows that for some $n$ sufficiently large we have

$$
\max \left\{\left|\nabla_{x} \varphi\left(\xi_{n}\right)\right|_{L},\left|\nabla_{y} \varphi\left(\xi_{n}\right)\right|_{M}\right\}<1 .
$$

Estimates in (6.38) also imply that

$$
\frac{\phi_{n}\left(\left|\nabla_{x} \varphi\left(\xi_{n}\right)\right|_{L}\right)}{\phi_{n}(1)\left|\nabla_{x} \varphi\left(\xi_{n}\right)\right|_{L}}\left|\Delta_{x} \varphi\left(\xi_{n}\right)\right| \leq\left|\nabla_{x} \varphi\left(\xi_{n}\right)\right|_{L}^{\phi_{n}^{-}-1}\left|\Delta_{x} \varphi\left(\xi_{n}\right)\right|,
$$

which in addition, if we have in mind that $\Delta_{x} \varphi\left(\xi_{n}\right) \rightarrow \Delta_{x} \varphi\left(\xi_{0}\right),\left|\nabla_{x} \varphi\left(\xi_{n}\right)\right|_{L} \rightarrow$ $\left|\nabla_{x} \varphi\left(\xi_{0}\right)\right|_{L}<1$ (hence for some $\varepsilon_{0}>0$ we have $\left|\nabla_{x} \varphi\left(\xi_{n}\right)\right|_{L} \leq 1-\varepsilon_{0}$ if $n$ large enough) and (1.3), indicates that the first term in (6.37) tends to zero. In a similar way,

$$
\lim _{n \rightarrow \infty} \frac{\psi_{n}\left(\left|\nabla_{y} \varphi\left(\xi_{n}\right)\right|_{L}\right)}{\psi_{n}(1)\left|\nabla_{y} \varphi\left(\xi_{n}\right)\right|_{L}} \Delta_{y} \varphi\left(\xi_{n}\right)=0
$$

Regarding the next term in (6.37) we note that

$$
\begin{aligned}
& \frac{\left|\nabla_{x} \varphi\left(\xi_{n}\right)\right|_{L} \phi_{n}^{\prime}\left(\left|\nabla_{x} \varphi\left(\xi_{n}\right)\right|_{L}\right)-\phi_{n}\left(\left|\nabla_{x} \varphi\left(\xi_{n}\right)\right|_{L}\right)}{\phi_{n}(1)\left|\nabla_{x} \varphi\left(\xi_{n}\right)\right|_{L}^{3}} \\
& =\frac{\phi_{n}\left(\left|\nabla_{x} \varphi\left(\xi_{n}\right)\right|_{L}\right)}{\phi_{n}(1)\left|\nabla_{x} \varphi\left(\xi_{n}\right)\right|_{L}^{3}}\left[\frac{\left|\nabla_{x} \varphi\left(\xi_{n}\right)\right|_{L} \phi_{n}^{\prime}\left(\left|\nabla_{x} \varphi\left(\xi_{n}\right)\right|_{L}\right)}{\phi_{n}\left(\left|\nabla_{x} \varphi\left(\xi_{n}\right)\right|_{L}\right)}-1\right] \\
& \leq \frac{\phi_{n}\left(\left|\nabla_{x} \varphi\left(\xi_{n}\right)\right|_{L}\right)}{\phi_{n}(1)} \frac{\phi_{n}^{+}-2}{\left|\nabla_{x} \varphi\left(\xi_{n}\right)\right|_{L}^{3}} \leq \frac{\left|\nabla_{x} \varphi\left(\xi_{n}\right)\right|_{L}^{\phi_{n}^{-}} \phi_{n}(1)}{\phi_{n}(1)} \frac{\phi_{n}^{+}-2}{\left|\nabla_{x} \varphi\left(\xi_{n}\right)\right|_{L}^{3}} \\
& \quad=\left(\left(\phi_{n}^{+}-2\right)^{\frac{1}{\phi_{n}^{-}-3}}\left|\nabla_{x} \varphi\left(\xi_{n}\right)\right|_{L}\right)^{\phi_{n}^{-}-3}
\end{aligned}
$$

where we have used (1.1). Now observe that

$$
\lim _{n \rightarrow \infty}\left(\phi_{n}^{+}-2\right)^{\frac{1}{\phi_{n}^{-}-3}}\left|\nabla_{x} \varphi\left(\xi_{n}\right)\right|_{L}=\left|\nabla_{x} \varphi\left(\xi_{0}\right)\right|_{L}<1,
$$

thus there exists $\varepsilon_{0}>0$ such that

$$
\left(\phi_{n}^{+}-2\right)^{\frac{1}{\phi_{n}^{-}-3}}\left|\nabla_{x} \varphi\left(\xi_{n}\right)\right|_{L} \leq 1-\varepsilon_{0}, \text { for } n \text { sufficiently large. }
$$

Therefore,

$$
\liminf _{n \rightarrow \infty} \frac{\left|\nabla_{x} \varphi\left(\xi_{n}\right)\right|_{L} \phi_{n}^{\prime}\left(\left|\nabla_{x} \varphi\left(\xi_{n}\right)\right|_{L}\right)-\phi_{n}\left(\left|\nabla_{x} \varphi\left(\xi_{n}\right)\right|_{L}\right)}{\phi_{n}(1)\left|\nabla_{x} \varphi\left(\xi_{n}\right)\right|_{L}^{3}} \leq \lim _{n \rightarrow \infty}\left(1-\varepsilon_{0}\right)^{\phi_{n}^{-}-3}=0,
$$

and similarly

$$
\liminf _{n \rightarrow \infty} \frac{\left|\nabla_{y} \varphi\left(\xi_{n}\right)\right|_{M} \psi_{n}^{\prime}\left(\left|\nabla_{y} \varphi\left(\xi_{n}\right)\right|_{M}\right)-\psi_{n}\left(\left|\nabla_{y} \varphi\left(\xi_{n}\right)\right|_{M}\right)}{\psi_{n}(1)\left|\nabla_{y} \varphi\left(\xi_{n}\right)\right|_{M}^{3}} \leq \lim _{n \rightarrow \infty}\left(1-\varepsilon_{0}\right)^{\psi_{n}^{-}-3}=0 .
$$

Clearly, $\Delta_{\infty, x} \varphi\left(\xi_{n}\right) \rightarrow \Delta_{\infty, x} \varphi\left(\xi_{0}\right)$ and $\Delta_{\infty, y} \varphi\left(\xi_{n}\right) \rightarrow \Delta_{\infty, y} \varphi\left(\xi_{0}\right)$. This shows that eventually the whole left hand side in (6.37) tends to zero, while the right hand side remains positive. We have reached the desired contradiction which shows that

$$
\max \left\{\left|\nabla_{x} \varphi\left(\xi_{0}\right)\right|_{L},\left|\nabla_{y} \varphi\left(\xi_{0}\right)\right|_{M}\right\} \geq 1 .
$$


Next, we show that $u_{\infty}$ is a viscosity subsolution. Fix $\xi_{0} \in \Omega$ and let $\varphi \in C^{2}(\Omega)$ be such that $u_{\infty}\left(\xi_{0}\right)=\varphi\left(\xi_{0}\right)$ and $u_{\infty}-\varphi$ has a local maximum at $\xi_{0}$. Let $\varepsilon>0$ be sufficiently small such that the set

$$
D_{\varepsilon}:=\{\xi \in \Omega:] \xi-\xi_{0}[1<\varepsilon\},
$$

satisfies $\overline{D_{\varepsilon}} \subset \Omega$. It is also clear that $D_{\varepsilon}$ is an open and convex subset of $\Omega$ for each $\varepsilon>0$ small enough. Let now $\eta_{L} \in \mathbb{R}^{L}$ be such that $\left|\eta_{L}\right|_{L}=1$. Define $\eta:=\left(\eta_{L}, 0_{M}\right) \in \mathbb{R}^{N}$, where $0_{M}$ stands for the null vector in $\mathbb{R}^{M}$. It is clear that for each $\varepsilon>0$ small enough we have

$$
]\left(\xi_{0} \pm \varepsilon \eta\right)-\xi_{0}\left[{ }_{1}=\right] \pm \varepsilon \eta\left[1=\varepsilon\left|\eta_{L}\right|_{L}=\varepsilon,\right.
$$

which implies that $\xi_{0} \pm \varepsilon \eta \in \partial D_{\varepsilon}$ and $]\left(\xi_{0} \pm \varepsilon \eta\right)-\xi_{0}\left[{ }_{1}=\varepsilon=\delta_{1}\left(\xi_{0}, \partial D_{\varepsilon}\right)\right.$ (and, thus, $\left.\xi_{0} \pm \varepsilon \eta \in A\left(\xi_{0}\right)\right)$ for each $\varepsilon>0$ small enough and for each $\eta$ constructed as above. By Lemma 6.1 and the fact that $\varphi$ is a test function such that $u_{\infty}\left(\xi_{0}\right)=\varphi\left(\xi_{0}\right)$ and $u_{\infty} \leq \varphi$ in $\overline{D_{\varepsilon}}$ for each $\varepsilon>0$ small enough we deduce that

$$
\varphi\left(\xi_{0}\right)=u_{\infty}\left(\xi_{0}\right)=\inf _{\theta \in A\left(\xi_{0}\right)}\left\{u_{\infty}(\theta)+\right] \xi_{0}-\theta[1\} \leq u_{\infty}\left(\xi_{0}-\varepsilon \eta\right)+\varepsilon \leq \varphi\left(\xi_{0}-\varepsilon \eta\right)+\varepsilon .
$$

Rearranging the previous expression, we get

$$
\frac{\varphi\left(\xi_{0}\right)-\varphi\left(\xi_{0}-\varepsilon \eta\right)}{\varepsilon} \leq 1
$$

Passing to the limit as $\varepsilon \rightarrow 0$ we deduce that

$$
\left\langle\nabla \varphi\left(\xi_{0}\right), \eta\right\rangle_{N} \leq 1
$$

or, equivalently

$$
\left\langle\nabla_{x} \varphi\left(\xi_{0}\right), \eta_{L}\right\rangle_{L} \leq 1, \quad \forall \eta_{L} \in \mathbb{R}^{L} \text { with }\left|\eta_{L}\right|_{L}=1,
$$

where we have denoted by $\langle\cdot, \cdot\rangle_{N}$ and $\langle\cdot, \cdot\rangle_{L}$ the scalar products on $\mathbb{R}^{N}$ and $\mathbb{R}^{L}$, respectively. Recalling the well-known fact that for each $x \in \mathbb{R}^{L}$ it holds true that

$$
|x|_{L}=\sup _{\left|\eta_{L}\right|_{L}=1}\left\langle x, \eta_{L}\right\rangle_{L},
$$

the above relation assures that

$$
\left|\nabla_{x} \varphi\left(\xi_{0}\right)\right|_{L} \leq 1
$$

Similar arguments can be used in order to show that

$$
\left|\nabla_{y} \varphi\left(\xi_{0}\right)\right|_{M} \leq 1 \text {. }
$$

Consequently we get

$$
\max \left\{\left|\nabla_{x} \varphi\left(\xi_{0}\right)\right|_{L},\left|\nabla_{y} \varphi\left(\xi_{0}\right)\right|_{M}\right\} \leq 1 .
$$

The proof of Theorem 6.1 is now complete.

Acknowledgements: M. Mihăilescu has been been partially supported by CNCSUEFISCDI Grant No. PN-III-P4-ID-PCE-2016-0035. M. Pérez-Llanos was supported by projects ANPCYT PICT2014-1771 and CONICET PIP 11220150100032CO (Argentine). 


\section{REFERENCES}

1. Robert A. Adams, Sobolev spaces, Academic Press [A subsidiary of Harcourt Brace Jovanovich, Publishers], New York-London, 1975, Pure and Applied Mathematics, Vol. 65. MR 0450957

2. Gunnar Aronsson, Extension of functions satisfying Lipschitz conditions, Ark. Mat. 6 (1967), 551-561 (1967). MR 0217665

3. Marino Belloni and Bernd Kawohl, The pseudo-p-Laplace eigenvalue problem and viscosity solutions as $p \rightarrow \infty$, ESAIM Control Optim. Calc. Var. 10 (2004), no. 1, 28-52. MR 2084254

4. T. Bhattacharya, E. DiBenedetto, and J. Manfredi, Limits as $p \rightarrow \infty$ of $\Delta_{p} u_{p}=f$ and related extremal problems, Rend. Sem. Mat. Univ. Politec. Torino (1989), no. Special Issue, 15-68 (1991), Some topics in nonlinear PDEs (Turin, 1989). MR 1155453

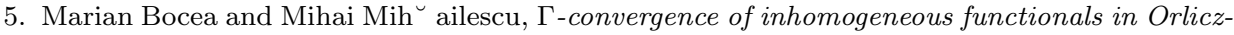
Sobolev spaces, Proc. Edinb. Math. Soc. (2) 58 (2015), no. 2, 287-303. MR 3341440

6. Marian Bocea and Mihai Mihăilescu, On a family of inhomogeneous torsional creep problems, Proc. Amer. Math. Soc. 145 (2017), no. 10, 4397-4409. MR 3690623

7. Andrea Braides, $\Gamma$-convergence for beginners, Oxford Lecture Series in Mathematics and its Applications, vol. 22, Oxford University Press, Oxford, 2002. MR 1968440

8. Haim Brezis, Functional analysis, Sobolev spaces and partial differential equations, Universitext, Springer, New York, 2011. MR 2759829

9. Philippe Clément, Ben de Pagter, Guido Sweers, and François de Thélin, Existence of solutions to a semilinear elliptic system through Orlicz-Sobolev spaces, Mediterr. J. Math. 1 (2004), no. 3, 241-267. MR 2094464

10. Michael G. Crandall, Hitoshi Ishii, and Pierre-Louis Lions, User's guide to viscosity solutions of second order partial differential equations, Bull. Amer. Math. Soc. (N.S.) 27 (1992), no. 1, 1-67. MR 1118699

11. Gianni Dal Maso, An introduction to $\Gamma$-convergence, Progress in Nonlinear Differential Equations and their Applications, vol. 8, Birkhäuser Boston, Inc., Boston, MA, 1993. MR 1201152

12. Ennio De Giorgi, Sulla convergenza di alcune successioni d'integrali del tipo dell'area, Rend. Mat. (6) 8 (1975), 277-294, Collection of articles dedicated to Mauro Picone on the occasion of his ninetieth birthday. MR 0375037

13. Ennio De Giorgi and Tullio Franzoni, Su un tipo di convergenza variazionale, Atti Accad. Naz. Lincei Rend. Cl. Sci. Fis. Mat. Natur. (8) 58 (1975), no. 6, 842-850. MR 0448194

14. Agnese Di Castro, Mayte Pérez-Llanos, and José Miguel Urbano, Limits of anisotropic and degenerate elliptic problems, Commun. Pure Appl. Anal. 11 (2012), no. 3, 1217-1229. MR 2968618

15. Nobuyoshi Fukagai, Masayuki Ito, and Kimiaki Narukawa, Positive solutions of quasilinear elliptic equations with critical Orlicz-Sobolev nonlinearity on $\mathbf{R}^{N}$, Funkcial. Ekvac. 49 (2006), no. 2, 235-267. MR 2271234

16. Toshihiro Ishibashi and Shigeaki Koike, On fully nonlinear PDEs derived from variational problems of $L^{p}$ norms, SIAM J. Math. Anal. 33 (2001), no. 3, 545-569. MR 1871409

17. Hitoshi Ishii, A simple, direct proof of uniqueness for solutions of the Hamilton-Jacobi equations of eikonal type, Proc. Amer. Math. Soc. 100 (1987), no. 2, 247-251. MR 884461

18. Robert Jensen, Uniqueness of Lipschitz extensions: minimizing the sup norm of the gradient, Arch. Rational Mech. Anal. 123 (1993), no. 1, 51-74. MR 1218686

19. Jürgen Jost and Xianqing Li-Jost, Calculus of variations, Cambridge Studies in Advanced Mathematics, vol. 64, Cambridge University Press, Cambridge, 1998. MR 1674720

20. Petri Juutinen, Minimization problems for Lipschitz functions via viscosity solutions, Ann. Acad. Sci. Fenn. Math. Diss. (1998), no. 115, 53, Dissertation, University of Jyväskulä, Jyväskulä, 1998. MR 1632063

21. Petri Juutinen, Peter Lindqvist, and Juan J. Manfredi, The $\infty$-eigenvalue problem, Arch. Ration. Mech. Anal. 148 (1999), no. 2, 89-105. MR 1716563

22. __ On the equivalence of viscosity solutions and weak solutions for a quasi-linear equation, SIAM J. Math. Anal. 33 (2001), no. 3, 699-717. MR 1871417

23. Bernhard Kawohl, On a family of torsional creep problems, J. Reine Angew. Math. 410 (1990), 1-22. MR 1068797

24. Gary M. Lieberman, The natural generalization of the natural conditions of Ladyzhenskaya and Ural'tseva for elliptic equations, Comm. Partial Differential Equations 16 (1991), no. 2-3, 311-361. MR 1104103 
25. Sandra Martí nez and Noemi Wolanski, A minimum problem with free boundary in Orlicz spaces, Adv. Math. 218 (2008), no. 6, 1914-1971. MR 2431665

26. L. E. Payne and G. A. Philippin, Some applications of the maximum principle in the problem of torsional creep, SIAM J. Appl. Math. 33 (1977), no. 3, 446-455. MR 0455738

27. Mayte Pérez-Llanos, Anisotropic variable exponent $(p(\cdot), q(\cdot))$-Laplacian with large exponents, Adv. Nonlinear Stud. 13 (2013), no. 4, 1003-1034. MR 3115150

28. Mayte Pérez-Llanos and Julio D. Rossi, The behaviour of the $p(x)$-Laplacian eigenvalue problem as $p(x) \rightarrow \infty$, J. Math. Anal. Appl. 363 (2010), no. 2, 502-511. MR 2564871

29. Mayte Perez-Llanos and Julio D. Rossi, The limit as $p(x) \rightarrow \infty$ of solutions to the inhomogeneous Dirichlet problem of the p(x)-Laplacian, Nonlinear Anal. 73 (2010), no. 7, 2027-2035. MR 2674182

Mihai Mihăilescu

Department of Mathematics

University of Craiova

200585 Craiova, Romania

AND

"Simion Stoilow" Institute of Mathematics of the Romanian Academy

010702 Bucharest, Romania.

mmihailes@yahoo.com

Mayte PÉrez-Llanos

iMAS-CONICET and Departamento e Matemática, FCEyN UBA,

Ciudad Universitaria, Pab 1, (1428),

Buenos Aires, ARGENTINA.

maytep@dm.uba.ar 